\title{
Asymptotic Behaviour of a Vibrating Membrane Model
}

\author{
M. Boella
}

\begin{abstract}
In this paper, we study the asymptotic behaviour of the solutions of an approximated model of the vibrating membrane. The results we give concern three different approximations; for each the boundedness of the energy and the presence of an absorbing set are proved, provided that the forcing term remains bounded in a suitable norm. The absorbing set is found via a known technique, while the boundedness of the energy is obtained by "ad hoc" calculations. Moreover, in one approximation, in the autonomous case, the existence of an attractor is proved.
\end{abstract}

Keywords: Absorbing set, vibrating membrane, asymptotic behaviour

AMS subject classification: 35B40, 35L70

\section{Introduction}

In this paper, we present some results regarding two approximate models of the nonlinear vibrating membrane equation

$$
v_{t t}-\operatorname{div}\left[\phi\left(\left(1+|\nabla v|^{2}\right)^{\frac{1}{2}}-\rho^{2}\right) \frac{\nabla v}{\left(1+|\nabla v|^{2}\right)^{\frac{1}{2}}}\right]-f=0
$$

where $\phi=\phi(s)$ represents the stress-strain law, $\rho$ is a constant depending on the initial stress with $0<\rho<1$ and $f$ is the external force. For the deduction of the model we refer to [6], where the reader will find also the proof of an existence and uniqueness theorem for the first approximate model.

As is pointed out in [6], no global existence theorem has been proved for the classical Cauchy-Dirichlet problem relative to equation (1). This fact suggested to modify the equation, by adding some small "fourth-order" term, thus recovering the "well-posedness" of the problem. This can be done in two different ways, using the terms $\gamma \Delta u_{t t}$ and $\eta \Delta^{2} u$, where the first takes

M. Boella: Politecnico di Milano, Dip. di Mat. "F. Brioschi", P.zza L. da Vinci 32, IT - 20133 Milano, Italia; mboella@crema.unimi.it

ISSN 0232-2064 / \$2.50 C Heldermann Verlag Berlin 
into account the small moment of inertia in every section of the membrane ( $\gamma=I E$, where $I$ is the moment of inertia and $E$ is Young modulus), the second is due to an approximation of the membrane with a thin plate, with a small $(\eta)$ bending moment; again, we refer to $[4,6]$ for further explanations and for the deduction respectively of the two approximations.

In our calculation, for the sake of simplicity, we choose a linear stressstrain relationship, $\phi(s)=K s$ with $K>0$ Hooke's constant; besides, as one of our aims is the search for an absorbing set, this suggests the introduction of a dissipative term $\delta u_{t}$.

Here below, in Section 1, we present the approximate models: first the model involving $\gamma \Delta u_{t t}$, together with its dissipative term $\gamma \delta \Delta u_{t}$ (we shall refer to it as the " $\gamma$ approximation"), then the model with $\eta \Delta^{2} u$ (the " $\eta$ approximation"), finally, the complete joint model (the " $\gamma, \eta$ approximation"), which combines both approximations.

In Section 2, we list all the tools we shall use in this paper, namely: notations, variational formulations of the approximate problems, energy and energy-type scalar products and norms.

In Section 3, we will give all the results for each model separately. We will list the theorems regarding the boundedness of energy for the solutions when $t \in \mathbb{R}^{+}$and the existence of an absorbing set, together with some minor results: the uniform continuity of the energy norms and the exponential decay of the energy in the autonomous case. Moreover, we state, for the $\gamma$ approximation, the existence of an attractor and, for the $\gamma, \eta$ approximation, the boundedness of energy when $t \in \mathbb{R}$.

The proofs are written in Section 4.

1.1 The approximations. Let now $\hat{u}$ be the solution relative to the $\gamma$ approximation, $\tilde{u}$ the solution relative to the $\eta$ approximation, and $u$ the solution relative to both approximations; the equations we shall study are the following (from now on, we shall denote by ${ }^{\prime}$ the time-derivative, while $\Omega$ denotes the domain covered by the membrane and $\mathbf{t}$ is the unit vector tangent to $\partial \Omega$.

The $\gamma$ approximation:

$$
\hat{u}^{\prime \prime}+\delta \hat{u}^{\prime}-K \Delta \hat{u}+K \rho^{2} \operatorname{div}\left(\frac{\nabla \hat{u}}{\left(1+|\nabla \hat{u}|^{2}\right)^{\frac{1}{2}}}\right)-\gamma \Delta \hat{u}^{\prime \prime}-\gamma \delta \Delta \hat{u}^{\prime}(t)=f
$$

with the corresponding boundary and initial conditions

$$
\begin{aligned}
& \left.\begin{array}{rl}
\hat{u}\left(t, x_{1}, x_{2}\right) & =0 \\
\nabla \hat{u}^{\prime}\left(t, x_{1}, x_{2}\right) \cdot \mathbf{t} & =0
\end{array}\right\} \quad\left(\left(x_{1}, x_{2}\right) \in \partial \Omega, t>0\right) \\
& \left.\begin{array}{rl}
\hat{u}\left(0, x_{1}, x_{2}\right) & =\alpha\left(x_{1}, x_{2}\right) \\
\hat{u}^{\prime}\left(0, x_{1}, x_{2}\right) & =\beta\left(x_{1}, x_{2}\right)
\end{array}\right\} \quad\left(\left(x_{1}, x_{2}\right) \in \Omega\right)
\end{aligned}
$$




\section{The $\eta$ approximation:}

$$
\tilde{u}^{\prime \prime}+\delta \tilde{u}^{\prime}-K \Delta \tilde{u}+K \rho^{2} \operatorname{div}\left(\frac{\nabla \tilde{u}}{\left(1+|\nabla \tilde{u}|^{2}\right)^{\frac{1}{2}}}\right)+\eta \Delta^{2} \tilde{u}=f
$$

with the corresponding boundary and initial conditions

$$
\begin{aligned}
& \left.\begin{array}{rl}
\tilde{u}\left(t, x_{1}, x_{2}\right) & =0 \\
\Delta \tilde{u}\left(t, x_{1}, x_{2}\right) & =0
\end{array}\right\} \quad\left(\left(x_{1}, x_{2}\right) \in \partial \Omega, t>0\right) \\
& \left.\begin{array}{rl}
\tilde{u}\left(0, x_{1}, x_{2}\right) & =\alpha\left(x_{1}, x_{2}\right) \\
\tilde{u}^{\prime}\left(0, x_{1}, x_{2}\right) & =\beta\left(x_{1}, x_{2}\right)
\end{array}\right\} \quad\left(\left(x_{1}, x_{2}\right) \in \Omega\right)
\end{aligned}
$$

The $\gamma, \eta$ approximation:

$$
u^{\prime \prime}+\delta u^{\prime}-K \Delta u+K \rho^{2} \operatorname{div}\left(\frac{\nabla u}{\left(1+|\nabla u|^{2}\right)^{\frac{1}{2}}}\right)-\gamma \Delta u^{\prime \prime}-\gamma \delta \Delta u^{\prime}(t)+\eta \Delta^{2} u=f
$$

with the corresponding boundary and initial conditions

$$
\begin{aligned}
& \left.\left.\begin{array}{rl}
u\left(t, x_{1}, x_{2}\right) & =0 \\
\nabla u^{\prime}\left(t, x_{1}, x_{2}\right) \cdot \mathbf{n} & =0 \\
\Delta u\left(t, x_{1}, x_{2}\right) & =0
\end{array}\right\} \quad\left(x_{1}, x_{2}\right) \in \partial \Omega, t>0\right) \\
& \left.\begin{array}{rl}
u\left(0, x_{1}, x_{2}\right) & =\alpha\left(x_{1}, x_{2}\right) \\
u^{\prime}\left(0, x_{1}, x_{2}\right) & =\beta\left(x_{1}, x_{2}\right)
\end{array}\right\} \quad\left(\left(x_{1}, x_{2}\right) \in \Omega\right) .
\end{aligned}
$$

\section{Definitions and notations}

Let $\Omega$ be a bounded open set in the $\left(x_{1}, x_{2}\right)$-plane, with a $C^{2}$-boundary (or an open convex polygon). We set $L^{2}=L^{2}(\Omega)$ and $H^{k}=H^{k}(\Omega)$. We shall use scalar products and norms of both scalar and vector functions (e.g. $\zeta$ and $\left.\nabla \zeta=\zeta_{x_{1}} \mathbf{i}+\zeta_{x_{2}} \mathbf{j}\right)$, with the following notations:

$$
\begin{aligned}
(\zeta, \chi) & =(\zeta, \chi)_{L^{2}} \\
(\nabla \zeta, \nabla \chi)_{L^{2} \times L^{2}} & =\left(\frac{\partial \zeta}{\partial x_{1}}, \frac{\partial \chi}{\partial x_{1}}\right)+\left(\frac{\partial \zeta}{\partial x_{2}}, \frac{\partial \chi}{\partial x_{2}}\right) \\
\|\zeta\|_{L^{2}}^{2} & =(\zeta, \zeta) \\
\|\nabla \zeta\|_{L^{2} \times L^{2}}^{2} & =(\nabla \zeta, \nabla \zeta)_{L^{2} \times L^{2}}
\end{aligned}
$$


We note that, as written above, we shall denote by $(\cdot, \cdot)$ the usual scalar product in $L^{2}$ when no confusion will be possible.

We shall denote by $C_{i}$ quantities depending only on physical and geometrical constants of the problem, while we shall use letters $A$ and $A_{i}$ to indicate constants depending also on initial conditions and/or on the known term $f$.

Finally, we denote by $\nu_{i}(\varepsilon)$ some (positive) continuous functions, vanishing as $\varepsilon \rightarrow 0$.

2.1 Auxiliary functions. It is useful, for our purposes, to define the auxiliary functions

$$
\begin{array}{r}
\mathbf{b}: \mathbb{R}^{2} \rightarrow \mathbb{R}^{2}, \quad \mathbf{b}\left(\xi_{1} \mathbf{i}+\xi_{2} \mathbf{j}\right)=K \rho^{2} \frac{\xi_{1} \mathbf{i}+\xi_{2} \mathbf{j}}{\sqrt{1+\xi_{1}^{2}+\xi_{2}^{2}}} \\
\psi: \mathbb{R} \rightarrow \mathbb{R}, \quad \psi(\xi)=K\left[1-\frac{\rho^{2}}{\sqrt{1+\xi}}\right]
\end{array}
$$

With these definitions, for example, equation (2) becomes

$$
\hat{u}^{\prime \prime}+\delta \hat{u}^{\prime}-\nabla\left(\psi\left(|\nabla \hat{u}|^{2}\right) \nabla \hat{u}\right)-\gamma \Delta \hat{u}^{\prime \prime}-\gamma \delta \Delta \hat{u}^{\prime}=f
$$

or

$$
\hat{u}^{\prime \prime}+\delta \hat{u}^{\prime}-\nabla(K \nabla \hat{u}-\mathbf{b}(\nabla \hat{u}))-\gamma \Delta \hat{u}^{\prime \prime}-\gamma \delta \Delta \hat{u}^{\prime}=f .
$$

If we set

$$
\Psi(\xi)=\int_{0}^{\xi} \psi(\sigma) d \sigma=K\left[\xi-\rho^{2}(\sqrt{1+\xi}-1)\right]
$$

it is quite easy to prove that

$$
\begin{aligned}
(-\operatorname{div} \psi & \left.\left(|\nabla \zeta(t)|^{2}\right) \nabla \zeta(t), \zeta^{\prime}(t)\right)_{L^{2}} \\
& =\left(\psi\left(|\nabla \zeta(t)|^{2}\right) \nabla \zeta(t), \nabla \zeta^{\prime}(t)\right)_{L^{2} \times L^{2}} \\
& =\frac{1}{2}\left(\psi\left(|\nabla \zeta(t)|^{2}\right), \frac{d}{d t}\left[|\nabla \zeta(t)|^{2}\right]\right)_{L^{2}} \\
& =\frac{1}{2} \frac{d}{d t}\left[\left(\Psi\left(|\nabla \zeta(t)|^{2}\right), 1\right)_{L^{2}}\right]
\end{aligned}
$$

and that the inequalities

$$
\begin{aligned}
& K\left(1-\rho^{2}\right)\|\nabla \zeta\|_{L^{2} \times L^{2}}^{2} \leq\left(\psi\left(|\nabla \zeta|^{2}\right) \nabla \zeta, \nabla \zeta\right)_{L^{2} \times L^{2}} \leq K\|\nabla \zeta\|_{L^{2} \times L^{2}}^{2} \\
& K\left(1-\rho^{2}\right)\|\nabla \zeta\|_{L^{2} \times L^{2}}^{2} \leq\left(\Psi\left(|\nabla \zeta|^{2}\right), 1\right) \leq K\|\nabla \zeta\|_{L^{2} \times L^{2}}^{2}
\end{aligned}
$$

hold. Moreover, from [6: Remark 3.2] we recall the property

$$
\left|\mathbf{b}\left(\mathbf{v}_{1}\right)-\mathbf{b}\left(\mathbf{v}_{2}\right)\right| \leq M\left|\mathbf{v}_{1}-\mathbf{v}_{2}\right|
$$


2.2 The smoothing operator $\boldsymbol{J}_{\boldsymbol{\mu}}$. In the sequel, we shall deal with scalar products not properly defined in $L^{2}$; as in [6], we introduce a smoothing operator, namely $J_{\mu}$.

Given $\mu>0$, we define the function $g_{\mu} \in C_{0}^{\infty}(\mathbb{R})$ by

$$
g_{\mu}(t)= \begin{cases}\frac{C}{\mu^{2}} \exp \left(\frac{\mu^{2}}{\mu^{2}-t^{2}}\right) & \text { for }|t|<\mu \\ 0 & \text { for }|t| \geq \mu\end{cases}
$$

with $C$ being such that $\int_{\mathbb{R}} g_{\mu}(t) d t=1$. We define now the operator $J_{\mu}$, acting on a function $\zeta(t, \mathbf{x})$ defined in $\mathbb{R} \times \Omega$, by

$$
J_{\mu} \zeta(t, \mathbf{x})=g_{\mu}(t) * \zeta(t, \mathbf{x})=\int_{+\infty}^{-\infty} g_{\mu}(t-\tau) \zeta(\tau, \mathbf{x}) d \tau
$$

provided, of course, the right-hand side of the above equation makes sense.

2.3 Variational formulation of the problems. For both approximations, we multiply the equation by a suitable test function $h$ and integrate from 0 to $t$.

The $\gamma$ approximation: In accordance with [6], we shall say that $\hat{u}$ is a solution in $[0, T] \times \Omega$ of problem (2)-(3), with $f \in L^{2}\left(0, T ; L^{2}\right), \alpha \in H^{2} \cap H_{0}^{1}$ and $\beta \in H_{0}^{1}$, if

$$
\left.\begin{array}{r}
W^{1, \infty}\left(0, T ; H^{2} \cap H_{0}^{1}\right) \ni \hat{u} \\
\hat{u}(0)=\alpha \\
\int_{0}^{t}\left\{-\left(\hat{u}^{\prime}(\tau), h^{\prime}(\tau)\right)+\delta\left(\hat{u}^{\prime}(\tau), h(\tau)\right)\right. \\
-\left(\nabla \psi\left(|\nabla \hat{u}(\tau)|^{2}\right) \nabla \hat{u}(\tau), h(\tau)\right)-\gamma\left(\nabla \hat{u}^{\prime}(\tau), \nabla h^{\prime}(\tau)\right) \\
\left.+\gamma \delta\left(\nabla \hat{u}^{\prime}(\tau), \nabla h(\tau)\right)-(f(\tau), h(\tau))\right\} d \tau \\
+\left(\hat{u}^{\prime}(t), h(t)\right)-(\beta, h(0)) \\
+\gamma\left(\nabla \hat{u}^{\prime}(t), \nabla h(t)\right)-\gamma(\nabla \beta, \nabla h(0))=0 \\
\text { a.e. in }[0, T], \forall h \in H^{1}\left(0, T ; H^{1}\right) .
\end{array}\right\}
$$

The $\eta$ approximation: We shall say that $\tilde{u}$ is a solution in $[0, T] \times \Omega$ of 
problem (4)-(5), with $f \in L^{2}\left(0, T ; L^{2}\right), \alpha \in H^{2} \cap H_{0}^{1}$ and $\beta \in L^{2}$, if

$$
\left.\begin{array}{r}
W^{1, \infty}\left(0, T ; L^{2}\right) \cap L^{\infty}\left(0, T ; H^{2} \cap H_{0}^{1}\right) \ni \tilde{u} \\
\tilde{u}(0)=\alpha \\
\int_{0}^{t}\left\{-\left(\tilde{u}^{\prime}(\tau), h^{\prime}(\tau)\right)+\delta\left(\tilde{u}^{\prime}(\tau), h(\tau)\right)\right. \\
-\left(\nabla \psi\left(|\nabla \tilde{u}(\tau)|^{2}\right) \nabla \tilde{u}(\tau), h(\tau)\right) \\
+\eta(\Delta \tilde{u}(\tau), \Delta h(\tau))-(f(\tau), h(\tau))\} d \tau \\
+\left(\tilde{u}^{\prime}(t), h(t)\right)-(\beta, h(0))=0
\end{array}\right\}
$$

a.e. in $[0, T], \forall h \in H^{1}\left(0, T ; L^{2}\right) \cap L^{2}\left(0, T ; H^{2} \cap H_{0}^{1}\right)$.

The $\gamma, \eta$ approximation: We shall say that $u$ is a solution in $[0, T] \times \Omega$ of problem (6)-(7), with $f \in L^{2}\left(0, T ; L^{2}\right), \alpha \in H^{2} \cap H_{0}^{1}$ and $\beta \in H_{0}^{1}$, if

$$
\left.\begin{array}{r}
W^{1, \infty}\left(0, T ; H^{2} \cap H_{0}^{1}\right) \ni u \\
u(0)=\alpha \\
\int_{0}^{t}\left\{-\left(u^{\prime}(\tau), h^{\prime}(\tau)\right)+\delta\left(u^{\prime}(\tau), h(\tau)\right)\right. \\
-\left(\nabla \psi\left(|\nabla u(\tau)|^{2}\right) \nabla u(\tau), h(\tau)\right)-\gamma\left(\nabla u^{\prime}(\tau), \nabla h^{\prime}(\tau)\right) \\
\left.+\gamma \delta\left(\nabla u^{\prime}(\tau), \nabla h(\tau)\right)+\eta(\Delta u(\tau), \Delta h(\tau))-(f(\tau), h(\tau))\right\} d \tau \\
+\left(u^{\prime}(t), h(t)\right)-(\beta, h(0))+\gamma\left(\nabla u^{\prime}(t), \nabla h(t)\right)-\gamma(\nabla \beta, \nabla h(0))=0 \\
\text { a.e. in }[0, T], \forall h \in H^{1}\left(0, T ; H^{1}\right) \cap L^{2}\left(0, T ; H^{2} \cap H_{0}^{1}\right) .
\end{array}\right\}
$$

2.4 Existence and uniqueness in $[0, T] \times \Omega$. We present these statements here, as they do not concern the long-time behaviour of the membrane. Their proofs are very similar to the ones in [6]. We shall give only a sketch about the continuous dependence from initial conditions.

Theorem 1. Let $f \in L^{2}\left(0, T ; L^{2}\right), \alpha \in H^{2} \cap H_{0}^{1}$ and $\beta \in H^{1}$. Then there exists in $Q$ a unique solution $\hat{u}$ of problem $(12), \hat{u} \in W^{1, \infty}\left(0, T ; H^{2} \cap H_{0}^{1}\right)$. Moreover, $\hat{u}$ depends continuosly on the data.

Theorem 2. Let $f \in L^{2}\left(0, T ; L^{2}\right), \alpha \in H^{2} \cap H_{0}^{1}$ and $\beta \in L^{2}$. Then there exists in $Q$ a unique solution $\tilde{u}$ of problem $(13), \tilde{u} \in W^{1, \infty}\left(0, T ; L^{2}\right) \cap$ $L^{\infty}\left(0, T ; H^{2} \cap H_{0}^{1}\right)$. Moreover, $\tilde{u}$ depends continuosly on the data.

Theorem 3. Let $f \in L^{2}\left(0, T ; L^{2}\right), \alpha \in H^{2} \cap H_{0}^{1}$ and $\beta \in H^{1}$. Then there exists in $Q$ a unique solution $u$ of problem (14), $u \in W^{1, \infty}\left(0, T ; H^{2} \cap H_{0}^{1}\right)$. Moreover, $u$ depends continuosly on the data. 
Remark 1. Aim of this paper is the study of the long-time behaviour of our membrane, so we want to emphasize now the hypoteses underlying all the theorems that will follow:

We set $\mathcal{V}=L_{\text {loc, },}^{2}\left(\mathbb{R}^{+}, L^{2}\right)$, and we shall always suppose $f \in \mathcal{V}$, setting

$$
\|f\|_{\mathcal{V}}^{2}=\sup _{t \in \mathbb{R}^{+}} \int_{t}^{t+1}\|f(\tau)\|_{L^{2}}^{2} d \tau .
$$

Remark 2. In order to study the long-time behaviour of the system, we need solutions defined for $t \in \mathbb{R}^{+}$. Indeed, we note that, if $f \in \mathcal{V}, \alpha \in H^{2} \cap H_{0}^{1}$ and $\beta \in H_{0}^{1}$, then it is possible to extend to $\mathbb{R}^{+} \times \Omega$ the solution $\hat{u}$ of problem (12); analogously, it is possible to extend to $\mathbb{R}^{+} \times \Omega$ the solutions $\tilde{u}$ of problem (13) and $u$ of problem (14).

In fact, we know (Theorem 3) that existence and uniqueness in $[0, k]$ of the solution, say $u_{k}(t)$, holds. We can now in the same way define the solution relative to $[0, k+1]$, say $u_{k+1}(t)$. It is clear that, for $t \in[0, k], u_{k}(t)=u_{k+1}(t)$, i.e. $u_{k+1}(t)$ is the extension to $[k, k+1]$ of $u_{k}(t)$. It is then possible to extend the solution for all $t>0$ and we shall denote this function by $u$.

2.5 Energy spaces and norms. In the sequel, we shall deal mostly with some energy-type norms, which we want to list here, each one in correspondence with its "energy space".

The $\gamma$ approximation: Let $\mathcal{H}_{\gamma}=\mathcal{H}_{\gamma}(\Omega)$ be the subset of $L^{2}$ defined as

$$
\mathcal{H}_{\gamma}=\left\{\left(\zeta(t), \zeta^{\prime}(t)\right): \zeta(t) \in H_{0}^{1}, \zeta^{\prime}(t) \in H_{0}^{1}\right\} .
$$

We shall define in $\mathcal{H}_{\gamma}$ the scalar product

$$
\begin{aligned}
(\zeta(t), \chi(t))_{\mathcal{H}_{\gamma}}= & \frac{1}{2}\left(\left(\zeta^{\prime}(t), \chi^{\prime}(t)\right)+K(\nabla \zeta(t), \nabla \chi(t))_{L^{2} \times L^{2}}\right. \\
& \left.+\gamma\left(\nabla \zeta^{\prime}(t), \nabla \chi^{\prime}(t)\right)_{L^{2} \times L^{2}}\right)
\end{aligned}
$$

and define then the "energy norm"

$$
E_{\gamma}(\zeta(t))=\frac{1}{2}\left(\left\|\zeta^{\prime}(t)\right\|_{L^{2}}^{2}+K\|\nabla \zeta(t)\|_{L^{2} \times L^{2}}^{2}+\gamma\left\|\nabla \zeta^{\prime}(t)\right\|_{L^{2} \times L^{2}}^{2}\right) .
$$

Moreover, if we set

$$
\begin{aligned}
& \mathcal{E}_{\gamma}(\zeta(t))=\left(\psi\left(|\nabla \zeta(t)|^{2}\right) \nabla \zeta(t), \nabla \zeta(t)\right)_{L^{2} \times L^{2}} \\
& \hat{E}_{\gamma}(\zeta(t))=\frac{1}{2}\left(\left\|\zeta^{\prime}(t)\right\|_{L^{2}}^{2}+\left(\Psi\left(|\nabla \zeta(t)|^{2}\right), 1\right)_{L^{2}}+\gamma\left\|\nabla \zeta^{\prime}(t)\right\|_{L^{2} \times L^{2}}^{2}\right)
\end{aligned}
$$


we can see that there is an equivalence between the three "energies" derived from these definitions. Indeed, thanks to (10), we can find three constants $C_{E i}$ such that

$$
\begin{aligned}
E_{\gamma}(\zeta(t)) & \leq C_{E 1} \hat{E}_{\gamma}(\zeta(t)) \\
& \leq C_{E 2}\left[\left\|\zeta^{\prime}(t)\right\|_{L^{2}}^{2}+\mathcal{E}_{\gamma}(\zeta(t))+\gamma\left\|\nabla \zeta^{\prime}(t)\right\|_{L^{2} \times L^{2}}^{2}\right] \\
& \leq C_{E 3} E_{\gamma}(\zeta(t)) .
\end{aligned}
$$

The $\eta$ approximation: Analogously to the previous subsection, we define the space

$$
\mathcal{H}_{\eta}=\left\{\left(\zeta(t), \zeta^{\prime}(t)\right) \mid \zeta(t) \in H_{0}^{1} \cap H^{2}, \zeta^{\prime}(t) \in L^{2}\right\}
$$

with the norm

$$
E_{\eta}(\zeta(t))=\frac{1}{2}\left(\left\|\zeta^{\prime}(t)\right\|_{L^{2}}^{2}+K\|\nabla \zeta(t)\|_{L^{2} \times L^{2}}^{2}+\eta\|\Delta \zeta(t)\|_{L^{2}}^{2}\right) .
$$

The $\gamma, \eta$ approximation: The energy space, for the joint approximation, is

$$
\mathcal{H}=\left\{\left(\zeta(t), \zeta^{\prime}(t)\right) \mid \zeta(t) \in H_{0}^{1} \cap H^{2}, \zeta^{\prime}(t) \in H_{0}^{1}\right\} .
$$

We note that $\mathcal{H}=\mathcal{H}_{\gamma} \cap \mathcal{H}_{\eta}$; the scalar product defined in $\mathcal{H}$ is

$$
\begin{aligned}
(\zeta(t), \chi(t))_{\mathcal{H}}= & \frac{1}{2}\left(\left(\zeta^{\prime}(t), \chi^{\prime}(t)\right)+K(\nabla \zeta(t), \nabla \chi(t))_{L^{2} \times L^{2}}\right. \\
& \left.+\gamma\left(\nabla \zeta^{\prime}(t), \nabla \chi^{\prime}(t)\right)_{L^{2} \times L^{2}}+\eta(\Delta \zeta(t), \Delta \chi(t))\right) .
\end{aligned}
$$

According to this scalar product, we define the (energy) norm by

$$
\begin{aligned}
E(\zeta(t))= & \frac{1}{2}\left(\left\|\zeta^{\prime}(t)\right\|_{L^{2}}^{2}+K\|\nabla \zeta(t)\|_{L^{2} \times L^{2}}^{2}\right. \\
& \left.+\gamma\left\|\nabla \zeta^{\prime}(t)\right\|_{L^{2} \times L^{2}}^{2}+\eta\|\Delta \zeta(t)\|_{L^{2}}^{2}\right) .
\end{aligned}
$$

2.6 The absorbing set. According to [3], we recall here the definition of "Absorbing set":

Definition 1. Given a space $H$, a set $B \subset H$ and an open set $U \supset B$, we say that $B$ is absorbing in $U$ if the orbit of any bounded set $B_{0} \subset U$ is contained in $B$, for all $t>\bar{t}\left(\bar{t}\right.$ may depend on $\left.B_{0}\right)$.

Our results about absorbing sets are obtained in the energy spaces, so the space $H$ cited in the definition of absorbing set will be regarded respectively as $\mathcal{H}_{\gamma}$ or $\mathcal{H}_{\eta}$ or $\mathcal{H}$, depending on the chosen approximation we are considering. 


\section{Results}

3.1 The $\gamma$ approximation. We recall that the $\gamma$ approximation is the unique among the ones discussed here which does not take into account spatial derivatives of higher order. In this case we can therefore prove a result (Theorem 7) regarding the existence of an attractor in the autonomous case.

Theorem 4 (Boundedness of energy for $t \in \mathbb{R}^{+}$). Let $f \in \mathcal{V}$ and let $\hat{u}(t)$ be the solution of problem (12) extended to $t \in \mathbb{R}^{+}$. Then there exists a constant $A$, depending on $f$ and on initial conditions,

$$
\begin{aligned}
& A=A\left(\|\beta\|_{L^{2}}^{2},\|\nabla \alpha\|_{L^{2} \times L^{2}}^{2},\|\nabla \beta\|_{L^{2} \times L^{2}}^{2},\|f\|_{\mathcal{V}}^{2}\right) \\
&=\max \left\{\left[C_{E 3} E_{\gamma}(\hat{u}(0))+C_{E 1} \frac{\|f\|_{\mathcal{V}}^{2}}{4 \delta}\right],\right. \\
& {\left.\left[C_{1}\|f\|_{\mathcal{V}}^{2}+\|f\|_{\mathcal{V}} \sqrt{C_{2}+C_{3}\|f\|_{\mathcal{V}}+C_{4}\|f\|_{\mathcal{V}}^{2}}\right]\right\}, }
\end{aligned}
$$

such that

$$
E_{\gamma}(\hat{u}(t)) \leq A \quad\left(t \in \mathbb{R}^{+}\right)
$$

Theorem 5 (Existence of an absorbing set). Let $f \in \mathcal{V}$ and let $\hat{u}(t)$ be the solution of problem (12) extended to $t \in \mathbb{R}^{+}$. Then there exist three constants $C_{1}, C_{2}, C_{3}>0$ such that

$$
E_{\gamma}(\hat{u}(t)) \leq C_{1}\|f\|_{\mathcal{V}}^{2}+C_{2} E_{\gamma}(\hat{u}(0)) e^{-C_{3} t} \quad\left(t \in \mathbb{R}^{+}\right) .
$$

As additional results we get

Theorem 6 (Uniform continuity of the energy norm for $t \in \mathbb{R}^{+}$). Let $f \in \mathcal{V}$ and let $\hat{u}(t)$ be the solution of problem (12) extended to $t \in \mathbb{R}^{+}$. Then the energy norm of $\hat{u}$ is uniformly continuous with respect to $t \in \mathbb{R}^{+}$, i.e.

$$
\left|E_{\gamma}(\hat{u}(t+\varepsilon))-E_{\gamma}(\hat{u}(t))\right| \leq \nu(\varepsilon) \quad\left(t \in \mathbb{R}^{+}\right) .
$$

As a corollary to Theorem 5, we can state the following

Corollary (Exponential decay). Let $f(t)=0$ and let $\hat{u}(t)$ be the solution of problem (12) extended to $t \in \mathbb{R}^{+}$. Then there exist two constants $C_{1}, C_{2}>0$ such that

$$
E_{\gamma}(\hat{u}(t)) \leq C_{1} E_{\gamma}(\hat{u}(0)) e^{-C_{2} t} \quad\left(t \in \mathbb{R}^{+}\right) .
$$

In the special case when the forcing term $f(t)$ does not depend on $t$ (the autonomous case), namely $f(t)=f_{0}$, one can prove the following result concerning the existence of an attractor: 
Theorem 7. Let $f_{0} \in L^{2}(\Omega)$. Then a solution $\hat{u}(t)$ to problem (12) tends to the solution $u$ of the autonomous equation $-K \Delta u+K \rho^{2} \operatorname{div}\left(\frac{\nabla u}{\left(1+|\nabla u|^{2}\right)^{1 / 2}}\right)=$ $f_{0}$.

3.2 The $\boldsymbol{\eta}$ approximation. The results presented here are completely analogous to those presented in Subsection 3.1.

Theorem 8 (Boundedness of the energy for $t \in \mathbb{R}^{+}$). Let $f \in \mathcal{V}$ and let $\tilde{u}(t)$ be the solution of problem (13) extended to $t \in \mathbb{R}^{+}$. Then there exists a constant $A$ depending on $f$ and on initial conditions,

$$
A=A\left(\|\beta\|_{L^{2}}^{2},\|\nabla \alpha\|_{L^{2} \times L^{2}}^{2},\|\Delta \alpha\|_{L^{2}}^{2},\|f\|_{\mathcal{V}}^{2}\right)
$$

such that

$$
E_{\eta}(\tilde{u}(t)) \leq A \quad\left(t \in \mathbb{R}^{+}\right) .
$$

Theorem 9 (Existence of an absorbing set). Let $f \in \mathcal{V}$ and let $\tilde{u}(t)$ be the solution of problem (13) extended to $t \in \mathbb{R}^{+}$. Then there exist three constants $C_{1}, C_{2}, C_{3}>0$ such that

$$
E_{\eta}(\tilde{u}(t)) \leq C_{1}\|f\|_{\mathcal{V}}^{2}+C_{2} E_{\eta}(\tilde{u}(0)) e^{-C_{3} t} \quad\left(t \in \mathbb{R}^{+}\right) .
$$

As additional results we get:

Theorem 10 (Uniform continuity of the energy norm for $t \in \mathbb{R}^{+}$). Let $f \in \mathcal{V}$ and let $\tilde{u}(t)$ be the solution of problem (13) extended to $t \in \mathbb{R}^{+}$. Then the energy norm of $\tilde{u}(t)$ is uniformly continuous with respect to $t \in \mathbb{R}^{+}$, i.e.

$$
\left|E_{\eta}(\tilde{u}(t+\varepsilon))-E_{\eta}(\tilde{u}(t))\right| \leq \nu(\varepsilon) \quad\left(t \in \mathbb{R}^{+}\right) .
$$

Corollary (Exponential decay). Let $f(t)=0$ and let $\tilde{u}(t)$ be the solution of problem (13) extended to $t \in \mathbb{R}^{+}$. Then there exist two constant $C_{1}, C_{2}>0$ such that

$$
E_{\eta}(\tilde{u}(t)) \leq C_{1} E_{\eta}(\tilde{u}(0)) e^{-C_{2} t} \quad\left(t \in \mathbb{R}^{+}\right)
$$

3.3 The $\gamma, \boldsymbol{\eta}$ approximation. In this subsection the results concerning joint approximation are collected together. Due to the simultaneous presence of a higher order derivative both in space and time, we can state (Theorem 13) global boundedness of the energy for $t \in \mathbb{R}$.

Theorem 11 (Boundedness of energy for $t \in \mathbb{R}^{+}$). Let $f \in \mathcal{V}$ and let $u(t)$ be the solution of problem (14) extended to $t \in \mathbb{R}^{+}$. Then there exists a constant $A$ depending on $f$ and on initial conditions

$$
A=A\left(\|\beta\|_{L^{2}}^{2},\|\nabla \alpha\|_{L^{2} \times L^{2}}^{2},\|\nabla \beta\|_{L^{2} \times L^{2}}^{2},\|\Delta \alpha\|_{L^{2}}^{2},\|f\|_{\mathcal{V}}^{2}\right)
$$


such that

$$
E(u(t)) \leq A \quad\left(t \in \mathbb{R}^{+}\right)
$$

Theorem 12 (Existence of an absorbing set). Let $f \in \mathcal{V}$ and let $u(t)$ be the solution of problem (14) extended to $t \in \mathbb{R}^{+}$. Then there exist three constants $C_{1}, C_{2}, C_{3}>0$ such that

$$
E(u(t)) \leq C_{1}\|f\|_{\mathcal{V}}^{2}+C_{2} E(u(0)) e^{-C_{3} t} \quad\left(t \in \mathbb{R}^{+}\right) .
$$

Theorem 13 (Boundedness of energy for $t \in \mathbb{R}$ ). Let $f \in \mathcal{V}$. Then there exist a solution $u(t)$, extendible for $t \in \mathbb{R}$, and a constant $A=A\left(\|f\|_{\mathcal{V}}^{2}\right)$, depending only on $f$, such that

$$
E(u(t)) \leq A \quad(t \in \mathbb{R}) .
$$

As additional results we get:

Theorem 14 (Uniform continuity of the energy norm for $t \in \mathbb{R}^{+}$). Let $f \in \mathcal{V}$ and let $u(t)$ be the solution of problem (14) extended to $t \in \mathbb{R}^{+}$. Then the energy norm of $u(t)$ is uniformly continuous with respect to $t \in \mathbb{R}^{+}$, i.e.

$$
|E(u(t+\varepsilon))-E(u(t))| \leq \nu(\varepsilon) \quad\left(t \in \mathbb{R}^{+}\right) .
$$

Corollary (Exponential decay). Let $f(t)=0$ and let $u(t)$ be the solution of problem (14) extended to $t \in \mathbb{R}^{+}$. Then there exist two constant $C_{1}, C_{2}>0$ such that

$$
E(u(t)) \leq C_{1} E(u(0)) e^{-C_{2} t} \quad\left(t \in \mathbb{R}^{+}\right)
$$

\section{Proofs}

In this section we will give the proofs of all our theorems.

Proofs of Theorems 1 - 3. The existence and uniqueness proofs of Theorems $1-3$ are similar to those in [6], so we omit to write them here in full. The continuous dependence from data can again be obtained using the technique illustrated in [6]. We write them for problem (12): if $\hat{u}_{1}$ and $\hat{u}_{2}$ are two solutions of problem (12), with each one its initial conditions, and $f_{1}$ and $f_{2}$ the forcing terms for $\hat{u}_{1}$ and $\hat{u}_{2}$, respectively. Setting $w=\hat{u}_{1}-\hat{u}_{2}$ and defining

$$
\mathcal{U}(t)=\frac{1}{2}\left(\left\|w^{\prime}(t)\right\|_{L^{2}}^{2}+K\|\nabla w(t)\|_{L^{2} \times L^{2}}^{2}+\gamma\left\|\nabla w^{\prime}(t)\right\|_{L^{2} \times L^{2}}^{2}\right)
$$

one can obtain

$$
\mathcal{U}(t) \leq \mathcal{U}(0)+C_{1} \int_{0}^{t}\left\|f_{1}(\tau)-f_{2}(\tau)\right\|_{L^{2}} d \tau+C_{2} \int_{0}^{t} \mathcal{U}(\tau) d \tau
$$

and the continuous dependence follows. From analogous calculations the continuous dependence from data in Theorems 2 and 3 can be deduced 
Proof of Theorem 4. Let $\left\{g_{i}\right\}$ be a basis in $H_{0}^{1}$, orthonormal in $L^{2}$, and let $\Pi_{n}$ be the projection operator on the subspace spanned by $\left\{g_{1}, \ldots, g_{n}\right\}$. Setting

$$
\hat{u}_{n}(t)=\sum_{i=1}^{n} \alpha_{n i}(t) g_{i}
$$

we can consider the system of ordinary differential equations in the unknowns $\alpha_{n i}$

$$
\begin{gathered}
\left(\hat{u}_{n}^{\prime \prime}(t), g_{i}\right) \\
-\left(\nabla \psi\left(\left|\nabla \hat{u}_{n}(t)\right|^{2}\right) \nabla \hat{u}_{n}(t), g_{i}\right)+\delta\left(\hat{u}_{n}^{\prime}(t), g_{i}\right) \\
-\gamma\left(\Delta \hat{u}_{n}^{\prime \prime}(t), g_{i}\right)-\gamma \delta\left(\Delta \hat{u}_{n}^{\prime}(t), g_{i}\right)-\left(f(t), g_{i}\right)=0 \quad(i=1, \ldots, n)
\end{gathered}
$$

together with the initial conditions

$$
\left.\begin{array}{l}
\hat{u}_{n}(0)=\Pi_{n} \alpha=\alpha_{n} \\
\hat{u}_{n}^{\prime}(0)=\Pi_{n} \beta=\beta_{n}
\end{array}\right\} .
$$

Multiplying (24) by $\alpha_{n i}^{\prime}(t)$ and adding, we get

$$
\begin{aligned}
& \left(\hat{u}_{n}^{\prime \prime}(t), \hat{u}_{n}^{\prime}(t)\right)-\left(\nabla \psi\left(\left|\nabla \hat{u}_{n}(t)\right|^{2}\right) \nabla \hat{u}_{n}(t), \hat{u}_{n}^{\prime}(t)\right) \\
& +\delta\left(\hat{u}_{n}^{\prime}(t), \hat{u}_{n}^{\prime}(t)\right)-\gamma\left(\Delta \hat{u}_{n}^{\prime \prime}(t), \hat{u}_{n}^{\prime}(t)\right) \\
& +\gamma \delta\left(\Delta \hat{u}_{n}^{\prime}(t), \hat{u}_{n}^{\prime}(t)\right)-\left(f(t), \hat{u}_{n}^{\prime}(t)\right)=0 .
\end{aligned}
$$

We can now integrate this equation from $\bar{t}$ to $\bar{t}+1$ and obtain

$$
\begin{aligned}
\int_{\bar{t}}^{\bar{t}+1}\left\{\left(\hat{u}_{n}^{\prime \prime}(\tau),\right.\right. & \left.\hat{u}_{n}^{\prime}(\tau)\right)+\left(\psi\left(\left|\nabla \hat{u}_{n}(\tau)\right|^{2}\right) \nabla \hat{u}_{n}(\tau), \nabla \hat{u}_{n}^{\prime}(\tau)\right)_{L^{2} \times L^{2}} \\
& +\delta\left(\hat{u}_{n}^{\prime}(\tau), \hat{u}_{n}^{\prime}(\tau)\right)+\gamma\left(\nabla \hat{u}_{n}^{\prime \prime}(\tau), \nabla \hat{u}_{n}^{\prime}(\tau)\right)_{L^{2} \times L^{2}} \\
+ & \left.\gamma \delta\left(\nabla \hat{u}_{n}^{\prime}(\tau), \nabla \hat{u}_{n}^{\prime}(\tau)\right)_{L^{2} \times L^{2}}-\left(f(\tau), \hat{u}_{n}^{\prime}(\tau)\right)\right\} d \tau=0
\end{aligned}
$$

that is, by (9),

$$
\begin{aligned}
\int_{\bar{t}}^{\bar{t}+1} & \left\{\frac{1}{2} \frac{d}{d t}\left[\left\|\hat{u}_{n}^{\prime}(\tau)\right\|_{L^{2}}^{2}+\gamma\left\|\nabla \hat{u}_{n}^{\prime}(\tau)\right\|_{L^{2} \times L^{2}}^{2}+\left(\Psi\left(\left|\nabla \hat{u}_{n}(\tau)\right|^{2}\right), 1\right)\right]\right\} d \tau \\
= & \int_{\bar{t}}^{\bar{t}+1}\left\{\left(f(\tau), \hat{u}_{n}^{\prime}(\tau)\right)-\delta\left\|\hat{u}_{n}^{\prime}(\tau)\right\|_{L^{2}}^{2}-\gamma \delta\left\|\nabla \hat{u}_{n}^{\prime}(\tau)\right\|_{L^{2} \times L^{2}}^{2}\right\} d \tau .
\end{aligned}
$$

Bearing in mind also (18) we can then write (26) as

$$
\begin{aligned}
& \hat{E}_{\gamma}\left(\hat{u}_{n}(\bar{t}+1)\right)-\hat{E}_{\gamma}\left(\hat{u}_{n}(\bar{t})\right) \\
& \quad=\int_{\bar{t}}^{\bar{t}+1}\left\{\left(f(\tau), \hat{u}_{n}^{\prime}(\tau)\right)-\delta\left\|\hat{u}_{n}^{\prime}(\tau)\right\|_{L^{2}}^{2}-\gamma \delta\left\|\nabla \hat{u}_{n}^{\prime}(\tau)\right\|_{L^{2} \times L^{2}}^{2}\right\} d \tau .
\end{aligned}
$$


Setting now $A_{1}=\frac{\|f\|_{v}^{2}}{\delta^{2}}$, we have only two cases: either

(a) $\int_{\bar{t}}^{\bar{t}+1}\left(\left\|\hat{u}_{n}^{\prime}(\tau)\right\|_{L^{2}}^{2}+\gamma\left\|\nabla \hat{u}_{n}^{\prime}(\tau)\right\|_{L^{2} \times L^{2}}^{2}\right) d \tau<A_{1}$

or at least one between $\hat{u}_{n}^{\prime}$ and $\nabla \hat{u}_{n}^{\prime}$ has a square norm greater than $A_{1}$ :

(b) $\int_{\bar{t}}^{\bar{t}+1}\left\|\hat{u}_{n}^{\prime}(\tau)\right\|_{L^{2}}^{2} d \tau \geq A_{1}$ or $\int_{\bar{t}}^{\bar{t}+1} \gamma\left\|\nabla \hat{u}_{n}^{\prime}(\tau)\right\|_{L^{2} \times L^{2}}^{2} d \tau \geq A_{1}$.

Case (b): To examine this case let us first suppose that $\int_{\bar{t}}^{\bar{t}+1}\left\|\hat{u}_{n}^{\prime}(\tau)\right\|_{L^{2}}^{2} d \tau$ $\geq A_{1}$. Then, independently from $\left\|\nabla \hat{u}_{n}^{\prime}(\tau)\right\|$, one can use the Schwartz inequality in (27) and get

$$
\begin{aligned}
& \int_{\bar{t}}^{\bar{t}+1}\left\{\left(f(\tau), \hat{u}_{n}^{\prime}(\tau)\right)-\delta\left\|\hat{u}_{n}^{\prime}(\tau)\right\|_{L^{2}}^{2}-\delta \gamma\left\|\nabla \hat{u}_{n}^{\prime}(\tau)\right\|_{L^{2} \times L^{2}}^{2}\right\} d \tau \\
& \quad \leq \int_{\bar{t}}^{\bar{t}+1}\left\{\left(\|f(\tau)\|_{L^{2}}-\delta\left\|\hat{u}_{n}^{\prime}(\tau)\right\|_{L^{2}}\right)\left\|\hat{u}_{n}^{\prime}(\tau)\right\|_{L^{2}}-\delta \gamma\left\|\nabla \hat{u}_{n}^{\prime}(\tau)\right\|_{L^{2} \times L^{2}}^{2}\right\} d \tau .
\end{aligned}
$$

Hence $\hat{E}_{\gamma}\left(\hat{u}_{n}(\bar{t}+1)\right)-\hat{E}_{\gamma}\left(\hat{u}_{n}(\bar{t})\right) \leq 0$.

Second, let us suppose that $\int_{\bar{t}}^{\bar{t}+1} \gamma\left\|\nabla \hat{u}_{n}^{\prime}(\tau)\right\|_{L^{2}}^{2} d \tau \geq A_{1}$. Independently from $\left\|\hat{u}_{n}^{\prime}(\tau)\right\|$ we can then write

$$
\begin{aligned}
& \int_{\bar{t}}^{\bar{t}+1}\left\{\left(f(\tau), \hat{u}_{n}^{\prime}(\tau)\right)-\delta\left\|\hat{u}_{n}^{\prime}(\tau)\right\|_{L^{2}}^{2}-\delta \gamma\left\|\nabla \hat{u}_{n}^{\prime}(\tau)\right\|_{L^{2} \times L^{2}}^{2}\right\} d \tau \\
& =\int_{\bar{t}}^{\bar{t}+1}\left\{\left(f(\tau), \hat{u}_{n}^{\prime}(\tau)\right)-\delta\left\|\hat{u}_{n}^{\prime}(\tau)\right\|_{L^{2}}^{2}-\left(\frac{1}{4 \delta}\right)\|f(\tau)\|_{L^{2}}^{2}\right. \\
& \left.\quad+\left(\frac{1}{4 \delta}\right)\|f(\tau)\|_{L^{2}}^{2}-\delta \gamma\left\|\nabla \hat{u}_{n}^{\prime}(\tau)\right\|_{L^{2} \times L^{2}}^{2}\right\} \\
& \quad \leq 0
\end{aligned}
$$

Hence again $\hat{E}_{\gamma}\left(\hat{u}_{n}(\bar{t}+1)\right)-\hat{E}_{\gamma}\left(\hat{u}_{n}(\bar{t})\right) \leq 0$.

Case (a): Obviously, then

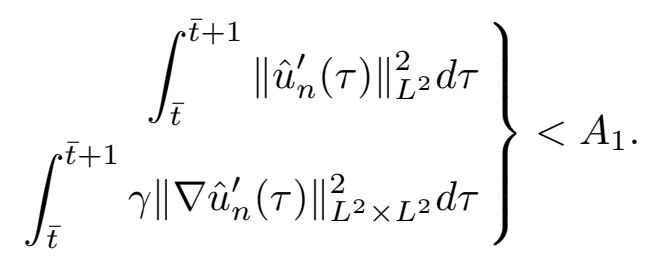

We can surely find $t^{\prime} \in\left(\bar{t}, \bar{t}+\frac{1}{4}\right)$ and $t^{\prime \prime} \in\left(\bar{t}+\frac{3}{4}, \bar{t}+1\right)$ so that

$$
\left.\begin{array}{r}
\left\|\hat{u}_{n}^{\prime}\left(t^{\prime}\right)\right\|_{L^{2}}^{2}+\left\|\nabla \hat{u}_{n}^{\prime}\left(t^{\prime}\right)\right\|_{L^{2} \times L^{2}}^{2} \\
\left\|\hat{u}_{n}^{\prime}\left(t^{\prime \prime}\right)\right\|_{L^{2}}^{2}+\left\|\nabla \hat{u}_{n}^{\prime}\left(t^{\prime \prime}\right)\right\|_{L^{2} \times L^{2}}^{2}
\end{array}\right\}<4 A_{1} .
$$


Moreover, for all $t^{*} \in(\bar{t}, \bar{t}+1)$ the inequalities

$$
\begin{aligned}
\left\|\hat{u}_{n}\left(t^{*}\right)\right\|_{L^{2}}^{2} & \leq 2\left\|\hat{u}_{n}(\bar{t})\right\|_{L^{2}}^{2}+2\left[\int_{\bar{t}}^{t *}\left\|\hat{u}_{n}^{\prime}(\tau)\right\|_{L^{2}} d \tau\right]^{2} \\
& \leq 2\left(\left\|\hat{u}_{n}(\bar{t})\right\|_{L^{2}}^{2}+\int_{\bar{t}}^{t *}\left\|\hat{u}_{n}^{\prime}(\tau)\right\|_{L^{2}}^{2} d \tau\right) \\
& \leq 2\left(\left\|\hat{u}_{n}(\bar{t})\right\|_{L^{2}}^{2}+A_{1}\right)
\end{aligned}
$$

hold. Hence we get

$$
\left\|\hat{u}_{n}\left(t^{*}\right)\right\|_{L^{2}} \leq \sqrt{2}\left[\left\|\hat{u}_{n}(\bar{t})\right\|_{L^{2}}^{2}+A_{1}\right]^{\frac{1}{2}}
$$

and similarly

$$
\left\|\nabla \hat{u}_{n}\left(t^{*}\right)\right\|_{L^{2} \times L^{2}} \leq \sqrt{2}\left[\left\|\nabla \hat{u}_{n}(\bar{t})\right\|_{L^{2} \times L^{2}}^{2}+A_{1}\right]^{\frac{1}{2}}
$$

Now, as we did in the beginning of the proof, we take the scalar product using $\hat{u}_{n}(t)$ instead of $\hat{u}_{n}^{\prime}(t)$ and then integrate the equation in $\left(t^{\prime}, t^{\prime \prime}\right)$ to get

$$
\begin{aligned}
\int_{t^{\prime}}^{t^{\prime \prime}}\left\{\left(\hat{u}_{n}^{\prime \prime}(\tau), \hat{u}_{n}(\tau)\right)-\left(\nabla\left(\psi\left(\left|\nabla \hat{u}_{n}(\tau)\right|^{2}\right) \nabla \hat{u}_{n}(\tau)\right), \hat{u}_{n}(\tau)\right)\right. \\
+\delta\left(\hat{u}_{n}^{\prime}(\tau), \hat{u}_{n}(\tau)\right)+\gamma\left(\nabla \hat{u}_{n}^{\prime \prime}(\tau), \nabla \hat{u}_{n}(\tau)\right)_{L^{2} \times L^{2}} \\
\left.+\gamma \delta\left(\nabla \hat{u}_{n}^{\prime}(\tau), \nabla \hat{u}_{n}(\tau)\right)_{L^{2} \times L^{2}}-\left(f(\tau), \hat{u}_{n}(\tau)\right)\right\} d \tau=0
\end{aligned}
$$

that is

$$
\begin{aligned}
\int_{t^{\prime}}^{t^{\prime \prime}} & \left\{\left(\psi\left(\left|\nabla \hat{u}_{n}(\tau)\right|^{2}\right) \nabla \hat{u}_{n}(\tau), \nabla \hat{u}_{n}(\tau)\right)_{L^{2} \times L^{2}}\right\} d \tau \\
= & \int_{t^{\prime}}^{t^{\prime \prime}}\left(\hat{u}_{n}^{\prime}(\tau), \hat{u}_{n}^{\prime}(\tau)\right) d \tau-\left[\left(\hat{u}_{n}^{\prime}(\tau), \hat{u}_{n}(\tau)\right)\right]_{t^{\prime}}^{t^{\prime \prime}}+\int_{t^{\prime}}^{t^{\prime \prime}}\left(f(\tau), \hat{u}_{n}(\tau)\right) d \tau \\
& +\gamma \int_{t^{\prime}}^{t^{\prime \prime}}\left(\nabla \hat{u}_{n}^{\prime}(\tau), \nabla \hat{u}_{n}^{\prime}(\tau)\right)_{L^{2} \times L^{2}} d \tau-\gamma\left[\left(\nabla \hat{u}_{n}^{\prime}(\tau), \nabla \hat{u}_{n}(\tau)\right)_{L^{2} \times L^{2}}\right]_{t^{\prime}}^{t^{\prime \prime}} \\
& -\delta \int_{t^{\prime}}^{t^{\prime \prime}}\left(\hat{u}_{n}^{\prime}(\tau), \hat{u}_{n}(\tau)\right) d \tau-\gamma \delta \int_{t^{\prime}}^{t^{\prime \prime}}\left(\nabla \hat{u}_{n}^{\prime}(\tau), \nabla \hat{u}_{n}(\tau)\right)_{L^{2} \times L^{2}} d \tau .
\end{aligned}
$$


Therefore, by (17), we obtain the inequality

$$
\begin{aligned}
\int_{t^{\prime}}^{t^{\prime \prime}} & \mathcal{E}_{\gamma}\left(\hat{u}_{n}(\tau)\right) d \tau \\
= & \int_{t^{\prime}}^{t^{\prime \prime}}\left\{\left(\psi\left(\left|\nabla \hat{u}_{n}(\tau)\right|^{2}\right) \nabla \hat{u}_{n}(\tau), \nabla \hat{u}_{n}(\tau)\right)_{L^{2} \times L^{2}}\right\} d \tau \\
\leq & \int_{t^{\prime}}^{t^{\prime \prime}}\left\|\hat{u}_{n}^{\prime}(\tau)\right\|_{L^{2}}^{2} d \tau+\left|\left[\left(\hat{u}_{n}^{\prime}(\tau), \hat{u}_{n}(\tau)\right)\right]_{t^{\prime}}^{t^{\prime \prime}}\right|+\left|\int_{t^{\prime}}^{t^{\prime \prime}}\left(f(\tau), \hat{u}_{n}(\tau)\right) d \tau\right| \\
& +\gamma \int_{t^{\prime}}^{t^{\prime \prime}}\left\|\nabla \hat{u}_{n}^{\prime}(\tau)\right\|_{L^{2} \times L^{2}}^{2} d \tau+\gamma\left|\left[\left(\nabla \hat{u}_{n}^{\prime}(\tau), \nabla \hat{u}_{n}(\tau)\right)_{L^{2} \times L^{2}}\right]_{t^{\prime}}^{t^{\prime \prime}}\right| \\
& +\left|\delta \int_{t^{\prime}}^{t^{\prime \prime}}\left(\hat{u}_{n}^{\prime}(\tau), \hat{u}_{n}(\tau)\right) d \tau\right|+\left|\gamma \delta \int_{t^{\prime}}^{t^{\prime \prime}}\left(\nabla \hat{u}_{n}^{\prime}(\tau), \nabla \hat{u}_{n}(\tau)\right)_{L^{2} \times L^{2}} d \tau\right|
\end{aligned}
$$

Now let us focalize on the terms in this upper limitation, and let us see how it is possible to bound every single item. We start considering the relation

$$
\begin{aligned}
\int_{t^{\prime}}^{t^{\prime \prime}} & \left(\left\|\hat{u}_{n}^{\prime}(\tau)\right\|_{L^{2}}^{2}+\left\|\nabla \hat{u}_{n}^{\prime}(\tau)\right\|_{L^{2} \times L^{2}}^{2}\right) d \tau \\
& \leq \int_{\bar{t}}^{\bar{t}+1}\left(\left\|\hat{u}_{n}^{\prime}(\tau)\right\|_{L^{2}}^{2}+\left\|\nabla \hat{u}_{n}^{\prime}(\tau)\right\|_{L^{2} \times L^{2}}^{2}\right) d \tau \\
& <A_{1} .
\end{aligned}
$$

Then we can write

$$
\begin{aligned}
\left|\left[\left(\hat{u}_{n}^{\prime}(\tau), \hat{u}_{n}(\tau)\right)\right]_{t^{\prime}}^{t^{\prime \prime}}\right| & \leq\left|\left(\hat{u}_{n}^{\prime}\left(t^{\prime \prime}\right), \hat{u}_{n}\left(t^{\prime \prime}\right)\right)\right|+\left|\left(\hat{u}_{n}^{\prime}\left(t^{\prime}\right), \hat{u}_{n}\left(t^{\prime}\right)\right)\right| \\
& \leq \sqrt{2}\left[\left\|\hat{u}_{n}(\bar{t})\right\|_{L^{2}}^{2}+A_{1}\right]^{\frac{1}{2}}\left(\left\|\hat{u}_{n}^{\prime}\left(t^{\prime \prime}\right)\right\|_{L^{2}}+\left\|\hat{u}_{n}^{\prime}\left(t^{\prime}\right)\right\|_{L^{2}}\right) \\
& \leq \sqrt{2}\left[\left\|\hat{u}_{n}(\bar{t})\right\|_{L^{2}}^{2}+A_{1}\right]^{\frac{1}{2}} 4 \sqrt{A_{1}} .
\end{aligned}
$$

In a completely analogous way we obtain

$$
\left|\left[\left(\nabla \hat{u}_{n}^{\prime}(\tau), \nabla \hat{u}_{n}(\tau)\right)_{L^{2} \times L^{2}}\right]_{t^{\prime}}^{t^{\prime \prime}}\right| \leq \sqrt{2}\left[\left\|\nabla \hat{u}_{n}(\bar{t})\right\|_{L^{2} \times L^{2}}^{2}+A_{1}\right]^{\frac{1}{2}} 4 \sqrt{A_{1}},
$$

but also

$$
\begin{aligned}
\left|\int_{t^{\prime}}^{t^{\prime \prime}}\left(f(\tau), \hat{u}_{n}(\tau)\right) d \tau\right| & \leq \int_{\bar{t}}^{\bar{t}+1}\left|\left(f(\tau), \hat{u}_{n}(\tau)\right)\right| d \tau \\
& \leq \sqrt{\|f\|_{\mathcal{V}}^{2}}\left(\int_{\bar{t}}^{\bar{t}+1}\left\|\hat{u}_{n}(\tau)\right\|_{L^{2}}^{2} d \tau\right)^{\frac{1}{2}} \\
& \leq \sqrt{2\|f\|_{\mathcal{V}}^{2}}\left[\left\|\hat{u}_{n}(\bar{t})\right\|_{L^{2}}^{2}+A_{1}\right]^{\frac{1}{2}}
\end{aligned}
$$


and, for the dissipative terms,

$$
\begin{aligned}
& \left|\delta \int_{t^{\prime}}^{t^{\prime \prime}}\left(\hat{u}_{n}^{\prime}(\tau), \hat{u}_{n}(\tau)\right) d \tau\right| \\
& \quad \leq \delta\left(\int_{t^{\prime}}^{t^{\prime \prime}}\left\|\hat{u}_{n}^{\prime}(\tau)\right\|_{L^{2}}^{2} d \tau\right)^{\frac{1}{2}}\left(\int_{t^{\prime}}^{t^{\prime \prime}}\left\|\hat{u}_{n}(\tau)\right\|_{L^{2}}^{2} d \tau\right)^{\frac{1}{2}} \\
& \quad \leq \delta \sqrt{2 A_{1}}\left[\left\|\hat{u}_{n}(\bar{t})\right\|_{L^{2}}^{2}+A_{1}\right]^{\frac{1}{2}}
\end{aligned}
$$

and

$$
\begin{aligned}
& \left|\gamma \delta \int_{t^{\prime}}^{t^{\prime \prime}}\left(\nabla \hat{u}_{n}^{\prime}(\tau), \nabla \hat{u}_{n}(\tau)\right) d \tau\right| \\
& \quad \leq \gamma \delta\left(\int_{t^{\prime}}^{t^{\prime \prime}}\left\|\nabla \hat{u}_{n}^{\prime}(\tau)\right\|_{L^{2} \times L^{2}}^{2} d \tau\right)^{\frac{1}{2}}\left(\int_{t^{\prime}}^{t^{\prime \prime}}\left\|\nabla \hat{u}_{n}(\tau)\right\|_{L^{2} \times L^{2}}^{2} d \tau\right)^{\frac{1}{2}} \\
& \quad \leq \gamma \delta \sqrt{2 A_{1}}\left[\left\|\nabla \hat{u}_{n}(\bar{t})\right\|_{L^{2} \times L^{2}}^{2}+A_{1}\right]^{\frac{1}{2}} .
\end{aligned}
$$

Hence, setting $A_{2}=(\delta+4) \sqrt{2 A_{1}}$ we obtain

$$
\begin{aligned}
\int_{t^{\prime}}^{t^{\prime \prime}} \mathcal{E}_{\gamma}\left(\hat{u}_{n}(\tau)\right) d \tau \leq & A_{1}+\left[\sqrt{2\|f\|_{\mathcal{V}}^{2}}+A_{2}\right]\left[\left\|\hat{u}_{n}(\bar{t})\right\|_{L^{2}}^{2}+A_{1}\right]^{\frac{1}{2}} \\
& +\gamma A_{2}\left[\left\|\nabla \hat{u}_{n}(\bar{t})\right\|_{L^{2} \times L^{2}}^{2}+A_{1}\right]^{\frac{1}{2}} .
\end{aligned}
$$

Bearing in mind that

$$
\int_{\bar{t}}^{\bar{t}+1}\left(\left\|\hat{u}_{n}^{\prime}(\tau)\right\|_{L^{2}}^{2}+\left\|\nabla \hat{u}_{n}^{\prime}(\tau)\right\|_{L^{2} \times L^{2}}^{2}\right) d \tau<A_{1}
$$

we get

$$
\begin{aligned}
\int_{t^{\prime}}^{t^{\prime \prime}} & \left(\mathcal{E}_{\gamma}\left(\hat{u}_{n}(\tau)\right)+\left\|\hat{u}_{n}^{\prime}(\tau)\right\|_{L^{2}}^{2}+\gamma\left\|\nabla \hat{u}_{n}^{\prime}(\tau)\right\|_{L^{2} \times L^{2}}^{2}\right) d \tau \\
\leq & 2 A_{1}+\left[\sqrt{2\|f\|_{\mathcal{V}}^{2}}+A_{2}\right]\left[\left\|\hat{u}_{n}(\bar{t})\right\|_{L^{2}}^{2}+A_{1}\right]^{\frac{1}{2}} \\
& +\gamma A_{2}\left[\left\|\nabla \hat{u}_{n}(\bar{t})\right\|_{L^{2} \times L^{2}}^{2}+A_{1}\right]^{\frac{1}{2}}
\end{aligned}
$$

Hence there exists $t^{*} \in\left[t^{\prime}, t^{\prime \prime}\right]$ so that

$$
\begin{aligned}
\mathcal{E}_{\gamma}\left(\hat{u}_{n}\left(t^{*}\right)\right)+\left\|\hat{u}_{n}^{\prime}\left(t^{*}\right)\right\|_{L^{2}}^{2}+\left\|\nabla \hat{u}_{n}^{\prime}\left(t^{*}\right)\right\|_{L^{2} \times L^{2}}^{2} & \\
\leq & 2\left(2 A_{1}+\left[\sqrt{2\|f\|_{\mathcal{V}}^{2}}+A_{2}\right]\left[\left\|\hat{u}_{n}(\bar{t})\right\|_{L^{2}}^{2}+A_{1}\right]^{\frac{1}{2}}\right. \\
& \left.+\gamma A_{2}\left[\left\|\nabla \hat{u}_{n}(\bar{t})\right\|_{L^{2} \times L^{2}}^{2}+A_{1}\right]^{\frac{1}{2}}\right) .
\end{aligned}
$$


By (19) we have

$$
\hat{E}_{\gamma}\left(\hat{u}_{n}\left(t^{*}\right)\right) \leq \frac{C_{E 2}}{C_{E 1}}\left[\mathcal{E}_{\gamma}\left(\hat{u}_{n}\left(t^{*}\right)\right)+\left\|\hat{u}_{n}\left(t^{*}\right)\right\|_{L^{2}}^{2}+\left\|\nabla \hat{u}_{n}\left(t^{*}\right)\right\|_{L^{2} \times L^{2}}^{2}\right] .
$$

Hence, by (28) there is a constant $C_{1}=2 \frac{C_{E 2}}{C_{E 1}}$ so that

$$
\begin{aligned}
\hat{E}_{\gamma}\left(\hat{u}_{n}\left(t^{*}\right)\right) \leq & C_{1}\left[2 A_{1}+\left[\sqrt{2\|f\|_{\mathcal{V}}^{2}}+A_{2}\right]\left[\left\|\hat{u}_{n}(\bar{t})\right\|_{L^{2}}^{2}+A_{1}\right]^{\frac{1}{2}}\right. \\
& \left.+\gamma A_{2}\left[\left\|\nabla \hat{u}_{n}(\bar{t})\right\|_{L^{2} \times L^{2}}^{2}+A_{1}\right]^{\frac{1}{2}}\right] .
\end{aligned}
$$

We now use (27) (with $t^{*}$ and $t$ instead of $\bar{t}$ and $\bar{t}+1$ ) to obtain $\hat{E}_{n}(t)$. Namely, for all $t \in[\bar{t}, \bar{t}+1]$ we have

$$
\begin{aligned}
\hat{E}_{\gamma}\left(\hat{u}_{n}(t)\right)= & \hat{E}_{\gamma}\left(\hat{u}_{n}\left(t^{*}\right)\right) \\
& +\int_{t^{*}}^{t}\left\{\left(f(\tau), \hat{u}_{n}^{\prime}(\tau)\right)-\delta\left\|\hat{u}_{n}^{\prime}(\tau)\right\|_{L^{2}}^{2}-\gamma \delta\left\|\nabla \hat{u}_{n}^{\prime}(\tau)\right\|_{L^{2} \times L^{2}}^{2}\right\} d \tau .
\end{aligned}
$$

Hence, letting $t=\bar{t}+1$ and bearing in mind (29) we get

$$
\begin{aligned}
\hat{E}_{\gamma}\left(\hat{u}_{n}(\bar{t}+1)\right) \\
\leq \hat{E}_{\gamma}\left(\hat{u}_{n}\left(t^{*}\right)\right)+\int_{t^{*}}^{\bar{t}+1}\left(f(\tau), \hat{u}_{n}^{\prime}(\tau)\right) d \tau \\
\leq \hat{E}_{\gamma}\left(\hat{u}_{n}\left(t^{*}\right)\right)+\left(\int_{t^{*}}^{\bar{t}+1}\|f(\tau)\|_{L^{2}}^{2} d \tau\right)^{\frac{1}{2}}\left(\int_{t^{*}}^{\bar{t}+1}\left\|\hat{u}_{n}^{\prime}(\tau)\right\|_{L^{2}}^{2} d \tau\right)^{\frac{1}{2}} \\
\leq C_{1}\left[2 A_{1}+\left[\sqrt{2\|f\|_{\mathcal{V}}^{2}}+A_{1}\right]\left[\left\|\hat{u}_{n}(\bar{t})\right\|_{L^{2}}^{2}+A_{1}\right]^{\frac{1}{2}}\right. \\
\left.\quad+\gamma A_{2}\left[\left\|\nabla \hat{u}_{n}(\bar{t})\right\|_{L^{2} \times L^{2}}^{2}+A_{1}\right]^{\frac{1}{2}}\right]+\sqrt{\|f\|_{\mathcal{V}}^{2} A_{1}} .
\end{aligned}
$$

Moreover, by (19) we have $E_{\gamma}\left(\hat{u}_{n}(\bar{t}+1)\right) \leq C_{E 1} \hat{E}_{\gamma}\left(\hat{u}_{n}(\bar{t}+1)\right)$. Setting $C_{2}=$ $C_{E 1} C_{1}=2 C_{E 2}$ we obtain

$$
\begin{aligned}
E_{\gamma}\left(\hat{u}_{n}(\bar{t}+1)\right) \leq & C_{2}\left[2 A_{1}+\left[\sqrt{2\|f\|_{\mathcal{V}}^{2}}+A_{2}\right]\left[\left\|\hat{u}_{n}(\bar{t})\right\|_{L^{2}}^{2}+A_{1}\right]^{\frac{1}{2}}\right. \\
& \left.+\gamma A_{2}\left[\left\|\nabla \hat{u}_{n}(\bar{t})\right\|_{L^{2} \times L^{2}}^{2}+A_{1}\right]^{\frac{1}{2}}\right]+\sqrt{\|f\|_{\mathcal{V}}^{2} A_{1}}
\end{aligned}
$$

By definition of $E_{\gamma}$, we have

$$
E_{\gamma}\left(\hat{u}_{n}(t)\right) \geq\left\{\begin{array}{l}
\left\|\hat{u}_{n}(t)\right\|_{L^{2}}^{2} \\
\left\|\nabla \hat{u}_{n}(t)\right\|_{L^{2} \times L^{2}}^{2}
\end{array}\right.
$$


for all $t$. Letting now

$$
\begin{aligned}
& A_{3}=\sqrt{\|f\|_{\mathcal{V}}^{2} A_{1}}+2 A_{1} C_{2} \\
& A_{4}=\sqrt{2\|f\|_{\mathcal{V}}^{2}}+(1+\gamma) A_{2}
\end{aligned}
$$

we have finally

$$
E_{\gamma}\left(\hat{u}_{n}(\bar{t}+1)\right) \leq A_{3}+A_{4} \sqrt{E_{\gamma}\left(\hat{u}_{n}(\bar{t})\right)+A_{1}} .
$$

If $E_{\gamma}\left(\hat{u}_{n}(\bar{t}+1)\right) \leq E_{\gamma}\left(\hat{u}_{n}(\bar{t})\right)$, the theorem is proved. If $E_{\gamma}\left(\hat{u}_{n}(\bar{t}+1)\right)>$ $E_{\gamma}\left(\hat{u}_{n}(\bar{t})\right)$, we substitute $E_{\gamma}\left(\hat{u}_{n}(\bar{t}+1)\right)$ in the square root of the above inequality and obtain

$$
E_{\gamma}\left(\hat{u}_{n}(\bar{t}+1)\right) \leq A_{3}+A_{4} \sqrt{E_{\gamma}\left(\hat{u}_{n}(\bar{t}+1)\right)+A_{1}} .
$$

Therefore, the inequality

$$
\left[E_{\gamma}\left(\hat{u}_{n}(\bar{t}+1)\right)\right]^{2}-2 A_{3} E_{\gamma}\left(\hat{u}_{n}(\bar{t}+1)\right)+A_{3}^{2} \leq A_{4}^{2}\left(E_{\gamma}\left(\hat{u}_{n}(\bar{t}+1)\right)+A_{1}\right)
$$

holds, that is

$$
\left[E_{\gamma}\left(\hat{u}_{n}(\bar{t}+1)\right)\right]^{2}-\left(2 A_{3}+A_{4}^{2}\right) E_{\gamma}\left(\hat{u}_{n}(\bar{t}+1)\right)+\left(A_{3}^{2}-A_{4}^{2} A_{1}\right) \leq 0 .
$$

This is a quadratic inequality, hence there exists a constant $A_{5}>0$ such that $E_{\gamma}\left(\hat{u}_{n}(\bar{t}+1)\right) \leq A_{5}$ from which it follows directly that

$$
A_{5}=C_{3}\|f\|_{\mathcal{V}}^{2}+\|f\|_{\mathcal{V}} \sqrt{C_{4}+C_{5}\|f\|_{\mathcal{V}}+C_{6}\|f\|_{\mathcal{V}}^{2}}
$$

Let now $0 \leq t \leq 1$. Thanks to the inequalities

$$
\begin{aligned}
\int_{0}^{t} & \left\{\left(f(\tau), \hat{u}_{n}^{\prime}(\tau)\right)-\delta\left\|\hat{u}_{n}^{\prime}(\tau)\right\|_{L^{2}}^{2}-\gamma \delta\left\|\nabla \hat{u}_{n}^{\prime}(\tau)\right\|_{L^{2} \times L^{2}}^{2}\right\} d \tau \\
= & \int_{0}^{t}\left\{\left[\left(f(\tau), \hat{u}_{n}^{\prime}(\tau)\right)-\delta\left\|\hat{u}_{n}^{\prime}(\tau)\right\|_{L^{2}}^{2}\right.\right. \\
& \left.\left.-\frac{1}{4 \delta}\|f(\tau)\|_{L^{2}}^{2}\right]+\frac{1}{4 \delta}\|f(\tau)\|_{L^{2}}^{2}-\gamma \delta\left\|\nabla \hat{u}_{n}^{\prime}(\tau)\right\|_{L^{2} \times L^{2}}^{2}\right\} d \tau \\
= & \int_{0}^{t}\left\{\frac{1}{4 \delta}\|f(\tau)\|_{L^{2}}^{2}-\left\|f(\tau)-\delta \hat{u}_{n}^{\prime}(\tau)\right\|_{L^{2}}^{2}-\gamma \delta\left\|\nabla \hat{u}_{n}^{\prime}(\tau)\right\|_{L^{2} \times L^{2}}^{2}\right\} d \tau \\
\leq & \frac{1}{4 \delta} \int_{0}^{1}\|f(\tau)\|_{L^{2}}^{2} d \tau
\end{aligned}
$$


we can write

$$
\hat{E}_{\gamma}\left(\hat{u}_{n}(t)\right) \leq \hat{E}_{\gamma}\left(\hat{u}_{n}(0)\right)+\frac{\|f\|_{\mathcal{V}}^{2}}{4 \delta}
$$

and, bearing in mind (19),

$$
E_{\gamma}\left(\hat{u}_{n}(t)\right) \leq A_{6}:=\left[C_{E 3} \hat{E}_{\gamma}\left(\hat{u}_{n}(0)\right)+C_{E 1} \frac{\|f\|_{\mathcal{V}}^{2}}{4 \delta}\right]
$$

We remind that

$$
E_{\gamma}\left(\hat{u}_{n}(0)\right)=\frac{1}{2}\left(\|\beta\|_{L^{2}}^{2}+\|\nabla \alpha\|_{L^{2} \times L^{2}}^{2}+\gamma\|\nabla \beta\|_{L^{2} \times L^{2}}^{2}\right) .
$$

Hence, letting $A_{7}=\max \left\{A_{6}, A_{5}\right\}$, e.g.

$$
\begin{aligned}
A_{7}=\max \{ & {\left[C_{E 3} E_{\gamma}\left(\hat{u}_{n}(0)\right)+C_{E 1} \frac{\|f\|_{\mathcal{V}}^{2}}{4 \delta}\right], } \\
& {\left.\left[C_{3}\|f\|_{\mathcal{V}}^{2}+\|f\|_{\mathcal{V}} \sqrt{C_{4}+C_{5}\|f\|_{\mathcal{V}}+C_{6}\|f\|_{\mathcal{V}}^{2}}\right]\right\}, }
\end{aligned}
$$

we obtain $E_{\gamma}\left(\hat{u}_{n}(t)\right) \leq A_{7}$.

Then for all $[0, k]$ it is possible to choose a sequence $\left\{\hat{u}_{n, k}(t)\right\}$ converging to $\hat{u}_{k}(t)$ weakly in $W^{1, \infty}\left(0, k ; H_{0}^{1}\right)$ and strongly in $L^{2}\left(0, k ; L^{2}\right)$. In [6] is shown that $\left\|\Delta \hat{u}_{n, k}^{\prime}(t)\right\| \leq M$ if $t \in[0, k]$, so $\psi\left(\mid \nabla \hat{u}_{n, k}(t)\right) \rightarrow \psi\left(\mid \nabla \hat{u}_{k}(t)\right)$. Moreover, $\left\{\hat{u}_{n, k}(t)\right\}$ is obtained as a subsequence from $\left\{\hat{u}_{n, k-1}(t)\right\}$ related to $[0, k-1]$. Letting $k \rightarrow \infty, \hat{u}(t)=\lim _{k} \hat{u}_{k}(t)$ is the solution of problem (12) extended to $\mathbb{R}^{+}$in the way we specified in Remark 2 . Then $E_{\gamma}(\hat{u}(t)) \leq A_{7}$ for all $t \in \mathbb{R}^{+}$

Proof of Theorem 5. We define $w_{n}(t, \mathbf{x})=\hat{u}_{n}^{\prime}(t, \mathbf{x})+\varepsilon \hat{u}_{n}(t, \mathbf{x})$, multiply (24) by $\alpha_{n i}^{\prime}(t)+\varepsilon \alpha_{n i}(t)$ and add to obtain

$$
\begin{array}{r}
\left(\hat{u}_{n}^{\prime \prime}(t), w_{n}(t)\right) \\
+\delta\left(\hat{u}_{n}^{\prime}(t), w_{n}(t)\right) \\
-\left(\nabla\left(\psi\left(\left|\nabla \hat{u}_{n}(t)\right|^{2}\right) \nabla \hat{u}_{n}(t)\right), w_{n}(t)\right) \\
+\gamma\left(\nabla \hat{u}_{n}^{\prime \prime}(t), \nabla w_{n}(t)\right)_{L^{2} \times L^{2}} \\
+\gamma \delta\left(\nabla \hat{u}_{n}^{\prime}(t), \nabla w_{n}(t)\right)_{L^{2} \times L^{2}}=\left(f(t), w_{n}(t)\right) .
\end{array}
$$

Let now us concentrate separately on each term of the above sum:

$$
\begin{aligned}
& \left(\hat{u}_{n}^{\prime \prime}(t), w_{n}(t)\right) \\
& \quad=\left(w_{n}^{\prime}(t)-\varepsilon \hat{u}_{n}^{\prime}(t), w_{n}(t)\right) \\
& \quad=\frac{1}{2} \frac{d}{d t}\left\|w_{n}(t)\right\|_{L^{2}}^{2}-\varepsilon\left(\hat{u}_{n}^{\prime}(t), w_{n}(t)\right)
\end{aligned}
$$




$$
\begin{aligned}
& =\frac{1}{2} \frac{d}{d t}\left\|w_{n}(t)\right\|_{L^{2}}^{2}-\varepsilon\left(w_{n}(t)-\varepsilon \hat{u}_{n}(t), w_{n}(t)\right) \\
& =\frac{1}{2} \frac{d}{d t}\left\|w_{n}(t)\right\|_{L^{2}}^{2}-\varepsilon\left\|w_{n}(t)\right\|_{L^{2}}^{2}+\varepsilon^{2}\left(\hat{u}_{n}(t), w_{n}(t)\right) \\
& \delta\left(\hat{u}_{n}^{\prime}(t), w_{n}(t)\right) \\
& =\delta\left(w_{n}(t)-\varepsilon \hat{u}_{n}(t), w_{n}(t)\right) \\
& =\delta\left\|w_{n}(t)\right\|_{L^{2}}^{2}-\varepsilon \delta\left(\hat{u}_{n}(t), w_{n}(t)\right) \\
& -\left(\nabla \psi\left(\left|\nabla \hat{u}_{n}(t)\right|^{2}\right) \nabla \hat{u}_{n}(t), w_{n}(t)\right) \\
& =\frac{1}{2} \frac{d}{d t}\left(\Psi\left(\left|\nabla \hat{u}_{n}(t)\right|^{2}\right), 1\right)+\varepsilon\left(\psi\left(\left|\nabla \hat{u}_{n}(t)\right|^{2}\right) \nabla \hat{u}_{n}(t), \nabla \hat{u}_{n}(t)\right)_{L^{2} \times L^{2}} \\
& \gamma\left(\nabla \hat{u}_{n}^{\prime \prime}(t), \nabla w_{n}(t)\right)_{L^{2} \times L^{2}} \\
& =\gamma\left(\nabla w_{n}^{\prime}(t)-\varepsilon \nabla \hat{u}_{n}^{\prime}(t), \nabla w_{n}(t)\right)_{L^{2} \times L^{2}} \\
& =\gamma \frac{1}{2} \frac{d}{d t}\left\|\nabla w_{n}(t)\right\|_{L^{2} \times L^{2}}^{2}-\varepsilon \gamma\left(\nabla \hat{u}_{n}^{\prime}(t), \nabla w_{n}(t)\right)_{L^{2} \times L^{2}} \\
& =\gamma \frac{1}{2} \frac{d}{d t}\left\|\nabla w_{n}(t)\right\|_{L^{2} \times L^{2}}^{2}-\varepsilon \gamma\left(\nabla\left(w_{n}(t)-\varepsilon \hat{u}_{n}(t)\right), \nabla w_{n}(t)\right)_{L^{2} \times L^{2}} \\
& =\gamma \frac{1}{2} \frac{d}{d t}\left\|\nabla w_{n}(t)\right\|_{L^{2} \times L^{2}}^{2}-\varepsilon \gamma\left\|\nabla w_{n}(t)\right\|_{L^{2} \times L^{2}}^{2} \\
& +\varepsilon^{2} \gamma\left(\nabla \hat{u}_{n}(t), \nabla w_{n}(t)\right)_{L^{2} \times L^{2}} \\
& \gamma \delta\left(\nabla \hat{u}_{n}^{\prime}(t), \nabla w_{n}(t)\right)_{L^{2} \times L^{2}} \\
& =\gamma \delta\left(\nabla w_{n}(t)-\varepsilon \nabla \hat{u}_{n}(t), \nabla w_{n}(t)\right)_{L^{2} \times L^{2}} \\
& =\gamma \delta\left\|\nabla w_{n}(t)\right\|_{L^{2} \times L^{2}}^{2}-\varepsilon \gamma \delta\left(\nabla \hat{u}_{n}(t), \nabla w_{n}(t)\right)_{L^{2} \times L^{2}} .
\end{aligned}
$$

Combining all these results, we obtain

$$
\begin{aligned}
\frac{1}{2} \frac{d}{d t}[ & \left.\left(\Psi\left(\left|\nabla \hat{u}_{n}(t)\right|^{2}\right), 1\right)+\left\|w_{n}(t)\right\|_{L^{2}}^{2}+\gamma\left\|\nabla w_{n}(t)\right\|_{L^{2} \times L^{2}}^{2}\right] \\
& +\varepsilon\left(\psi\left(\left|\nabla \hat{u}_{n}(t)\right|^{2}\right) \nabla \hat{u}_{n}(t), \nabla \hat{u}_{n}(t)\right)_{L^{2} \times L^{2}} \\
& +(\delta-\varepsilon)\left[\left\|w_{n}(t)\right\|_{L^{2}}^{2}+\gamma\left\|\nabla w_{n}(t)\right\|_{L^{2} \times L^{2}}^{2}\right] \\
& +\varepsilon(\varepsilon-\delta)\left[\left(\hat{u}_{n}(t), w_{n}(t)\right)+\gamma\left(\nabla \hat{u}_{n}(t), \nabla w_{n}(t)\right)_{L^{2} \times L^{2}}\right]=\left(f(t), w_{n}(t)\right) .
\end{aligned}
$$

Using now Young's inequality on $\left(\hat{u}_{n}(t), w_{n}(t)\right)$ and $\left(\nabla \hat{u}_{n}(t), \nabla w_{n}(t)\right)_{L^{2} \times L^{2}}$ we may write

$$
\begin{aligned}
\frac{1}{2} \frac{d}{d t}[ & \left.\left(\Psi\left(\left|\nabla \hat{u}_{n}(t)\right|^{2}\right), 1\right)+\left\|w_{n}(t)\right\|_{L^{2}}^{2}+\gamma\left\|\nabla w_{n}(t)\right\|_{L^{2} \times L^{2}}^{2}\right] \\
& +\varepsilon\left(\psi\left(\left|\nabla \hat{u}_{n}(t)\right|^{2}\right) \nabla \hat{u}_{n}(t), \nabla \hat{u}_{n}(t)\right)_{L^{2} \times L^{2}} \\
& +(\delta-\varepsilon)\left[\left\|w_{n}(t)\right\|_{L^{2}}^{2}+\gamma\left\|\nabla w_{n}(t)\right\|_{L^{2} \times L^{2}}^{2}\right]
\end{aligned}
$$




$$
\begin{aligned}
& +\varepsilon(\varepsilon-\delta)\left(C_{1}\left\|\hat{u}_{n}(t)\right\|_{L^{2}}^{2}+C_{2}\left\|w_{n}(t)\right\|_{L^{2}}^{2}\right) \\
& +\varepsilon(\varepsilon-\delta) \gamma\left(C_{3}\left\|\nabla \hat{u}_{n}(t)\right\|_{L^{2} \times L^{2}}^{2}+C_{4}\left\|\nabla w_{n}(t)\right\|_{L^{2} \times L^{2}}^{2}\right) \leq\left(f(t), w_{n}(t)\right) .
\end{aligned}
$$

Let us observe that, thanks to the two inequalities (10), there is a constant $C_{5}$ such that

$$
C_{5}\left(\Psi\left(\left|\nabla \hat{u}_{n}(t)\right|^{2}\right), 1\right) \leq\left(\psi\left(\left|\nabla \hat{u}_{n}(t)\right|^{2}\right) \nabla \hat{u}_{n}(t), \nabla \hat{u}_{n}(t)\right)_{L^{2} \times L^{2}} .
$$

We can then choose constants $C_{1}$ and $C_{3}$ both sufficiently small to find a suitable constant $C_{6}$ such that

$$
\begin{aligned}
\varepsilon C_{6}\left(\Psi\left(\left|\nabla \hat{u}_{n}(t)\right|^{2}\right), 1\right) \leq & \varepsilon\left(\psi\left(\left|\nabla \hat{u}_{n}(t)\right|^{2}\right) \nabla \hat{u}_{n}(t), \nabla \hat{u}_{n}(t)\right)_{L^{2} \times L^{2}} \\
& +\varepsilon(\varepsilon-\delta)\left(C_{1}\left\|\hat{u}_{n}(t)\right\|_{L^{2}}^{2}+C_{3} \gamma\left\|\nabla \hat{u}_{n}(t)\right\|_{L^{2} \times L^{2}}^{2}\right) .
\end{aligned}
$$

Therefore we obtain

$$
\begin{aligned}
\frac{1}{2} \frac{d}{d t} & {\left[\left(\Psi\left(\left|\nabla \hat{u}_{n}(t)\right|^{2}\right), 1\right)+\left\|w_{n}(t)\right\|_{L^{2}}^{2}+\gamma\left\|\nabla w_{n}(t)\right\|_{L^{2} \times L^{2}}^{2}\right] } \\
& +\varepsilon C_{6}\left(\Psi\left(\left|\nabla \hat{u}_{n}(t)\right|^{2}\right), 1\right) \\
& +\left[(\delta-\varepsilon)+\varepsilon(\varepsilon-\delta) C_{2}\right]\left\|w_{n}(t)\right\|_{L^{2}}^{2} \\
& +\gamma\left[(\delta-\varepsilon)+\varepsilon(\varepsilon-\delta) C_{4}\right]\left\|\nabla w_{n}(t)\right\|_{L^{2} \times L^{2}}^{2} \leq\|f(t)\|_{L^{2}}\left\|w_{n}(t)\right\|_{L^{2}} .
\end{aligned}
$$

If we now set

$$
\mathcal{U}_{n}(t)=\frac{1}{2}\left[\left(\Psi\left(\left|\nabla \hat{u}_{n}(t)\right|^{2}\right), 1\right)+\left\|w_{n}(t)\right\|_{L^{2}}^{2}+\gamma\left\|\nabla w_{n}(t)\right\|_{L^{2} \times L^{2}}^{2}\right],
$$

there exist two constants $C_{7}$ and $C_{8}$ such that

$$
\frac{d}{d t} \mathcal{U}_{n}(t)+C_{7} \mathcal{U}_{n}(t) \leq C_{8}\|f(t)\|_{L^{2}} \sqrt{\mathcal{U}_{n}(t)} .
$$

The Gronwall lemma then implies that, for all $t>0$,

$$
\mathcal{U}_{n}(t) \leq \frac{1}{e^{C_{7} t}}\left[\mathcal{U}_{n}(0)+\int_{0}^{t} e^{C_{7} \tau} C_{8}\|f(\tau)\|_{L^{2}} \sqrt{\mathcal{U}_{n}(\tau)} d \tau\right]
$$

If we now apply [5: Lemma A.1] ${ }^{1)}$, letting $k_{0}=0, k_{1}=\mathcal{U}_{n}(0), \delta=C_{7}$, $\phi(t)=\sqrt{\mathcal{U}_{n}(t)}$ and $m(t)=C_{8}\|f(t)\|_{L^{2}}$, we obtain

$$
\mathcal{U}_{n}(t) \leq 2 \mathcal{U}_{n}(0) e^{-C_{7} t}+\left(C_{8}\right)^{2}\left(\int_{0}^{t}\|f(s)\|_{L^{2}} e^{-\frac{C_{7}}{2}(t-s)} d s\right)^{2} .
$$

1) This lemma is stated as follows: Let $k_{0}, k_{1} \geq 0, m \in L_{l o c}^{1}\left(\mathbb{R}^{+}\right)$with $m \geq 0$ a.e. and $\phi \in C\left(\mathbb{R}^{+}\right)$with $\phi \geq 0$. If $\phi(t)^{2} \leq k_{0}+k_{1} e^{-\delta t}+\int_{0}^{t} m(s) \phi(s) e^{-\delta(t-s)} d s$ for any $t>0$ and some $\delta>0$, then $\phi(t)^{2} \leq 2 k_{0}+2 k_{1} e^{-\delta t}+\int_{0}^{t} m(s) e^{-\frac{\delta}{2}(t-s)} d s^{2}$ for any $t>0$. 
Let us now evaluate the above integral. First, we observe that

$$
\int_{k}^{k+1}\|f(s)\|_{L^{2}} d s=\int_{k}^{k+1}\|f(s)\|_{L^{2}} \cdot 1 d s \leq\|f\|_{L^{2}\left(k, k+1 ; L^{2}\right)}
$$

which implies $\sup _{k \in \mathbb{N}} \int_{k}^{k+1}\|f(s)\|_{L^{2}} d s \leq\|f\|_{\mathcal{V}}$. Then, letting $n \in \mathbb{N}$ such that $n-1<t \leq n$, we obtain

$$
\begin{aligned}
\left(\int_{0}^{t}\|f(s)\|_{L^{2}} e^{\frac{C_{7}}{2} s} d s\right)^{2} & \leq\left(\sum_{k=1}^{n} e^{\frac{C_{7}}{2} k} \int_{k-1}^{k}\|f(s)\|_{L^{2}} d s\right)^{2} \\
& \leq\|f\|_{\mathcal{V}}^{2}\left(\frac{1-e^{\frac{C_{7}}{2}(n+1)}}{1-e^{\frac{C_{7}}{2}}}\right)^{2} \\
& \leq\|f\|_{\mathcal{V}}^{2}\left(\frac{1-e^{\frac{C_{7}}{2}(t+2)}}{1-e^{\frac{C_{7}}{2}}}\right)^{2} \\
& \leq e^{C_{7} t}\|f\|_{\mathcal{V}}^{2} \frac{e^{2 C_{7}}}{\left(1-e^{\frac{C_{7}}{2}}\right)^{2}}
\end{aligned}
$$

Therefore, setting $C_{9}=\left(\frac{C_{8} e^{C_{7}}}{1-e^{C_{7} / 2}}\right)^{2}$ we have

$$
\mathcal{U}_{n}(t) \leq 2 \mathcal{U}_{n}(0) e^{-C_{7} t}+C_{9}\|f\|_{\mathcal{V}}^{2}
$$

observing that, with $w_{n}=\hat{u}_{n}^{\prime}+\varepsilon \hat{u}_{n}$,

$$
\begin{aligned}
2 \mathcal{U}_{n}(t)= & \left(\Psi\left(\left|\nabla \hat{u}_{n}(t)\right|^{2}\right), 1\right)+\left\|w_{n}(t)\right\|_{L^{2}}^{2}+\gamma\left\|\nabla w_{n}(t)\right\|_{L^{2} \times L^{2}}^{2} \\
= & \left(\Psi\left(\left|\nabla \hat{u}_{n}(t)\right|^{2}\right), 1\right)+\left\|\hat{u}_{n}^{\prime}(t)+\varepsilon \hat{u}_{n}(t)\right\|_{L^{2}}^{2} \\
& +\gamma\left\|\nabla \hat{u}_{n}^{\prime}(t)+\varepsilon \nabla \hat{u}_{n}(t)\right\|_{L^{2} \times L^{2}}^{2} \\
\geq & \left(\Psi\left(\left|\nabla \hat{u}_{n}(t)\right|^{2}\right), 1\right)+\left\|\hat{u}_{n}^{\prime}(t)\right\|_{L^{2}}^{2}+\gamma\left\|\nabla \hat{u}_{n}^{\prime}(t)\right\|_{L^{2} \times L^{2}}^{2} \\
& -2 \varepsilon\left\|\hat{u}_{n}^{\prime}(t)\right\|_{L^{2}}\left\|\hat{u}_{n}(t)\right\|_{L^{2}}-2 \gamma \varepsilon\left\|\nabla \hat{u}_{n}^{\prime}(t)\right\|_{L^{2} \times L^{2}}\left\|\nabla \hat{u}_{n}(t)\right\|_{L^{2} \times L^{2}} \\
\geq & \left(\Psi\left(\left|\nabla \hat{u}_{n}(t)\right|^{2}\right), 1\right)+(1-\varepsilon)\left\|\hat{u}_{n}^{\prime}(t)\right\|_{L^{2}}^{2} \\
& +\gamma(1-\varepsilon)\left\|\nabla \hat{u}_{n}^{\prime}(t)\right\|_{L^{2} \times L^{2}}^{2}-\varepsilon\left\|\hat{u}_{n}(t)\right\|_{L^{2}}^{2}-\gamma \varepsilon\left\|\nabla \hat{u}_{n}(t)\right\|_{L^{2} \times L^{2}}^{2} .
\end{aligned}
$$

We can then choose $\varepsilon$ sufficiently small to have

$$
\left(\Psi\left(\left|\nabla \hat{u}_{n}(t)\right|^{2}\right), 1\right)+(1-\varepsilon)\left\|\hat{u}_{n}^{\prime}(t)\right\|_{L^{2}}^{2}+\gamma(1-\varepsilon)\left\|\nabla \hat{u}_{n}^{\prime}(t)\right\|_{L^{2} \times L^{2}}^{2} \leq 2 \mathcal{U}_{n}(t) .
$$


Besides, we can find a constant $C_{10}$ such that

$$
\begin{aligned}
2 \mathcal{U}_{n}(0)= & \left(\Psi\left(\left|\nabla \hat{u}_{n}(0)\right|^{2}\right), 1\right)+\|w(0)\|_{L^{2}}^{2}+\gamma\|\nabla w(0)\|_{L^{2} \times L^{2}}^{2} \\
= & \left(\Psi\left(\left|\nabla \hat{u}_{n}(0)\right|^{2}\right), 1\right)+\left\|\hat{u}_{n}^{\prime}(0)+\varepsilon \hat{u}_{n}(0)\right\|_{L^{2}}^{2} \\
& +\gamma\left\|\nabla \hat{u}_{n}^{\prime}(0)+\varepsilon \nabla \hat{u}_{n}(0)\right\|_{L^{2} \times L^{2}}^{2} \\
\leq & \left(\Psi\left(\left|\nabla \hat{u}_{n}(0)\right|^{2}\right), 1\right) \\
& +(1+2 \varepsilon)\left\|\hat{u}_{n}^{\prime}(0)\right\|_{L^{2}}^{2}+\gamma(1+2 \varepsilon)\left\|\nabla \hat{u}_{n}^{\prime}(0)\right\|_{L^{2} \times L^{2}}^{2} \\
& +\left(2 \varepsilon+\varepsilon^{2}\right)\left\|\hat{u}_{n}^{\prime}(0)\right\|_{L^{2}}^{2}+\gamma\left(2 \varepsilon+\varepsilon^{2}\right)\left\|\nabla \hat{u}_{n}^{\prime}(0)\right\|_{L^{2}}^{2} \\
\leq & C_{10}\left(\left(\Psi\left(\left|\nabla \hat{u}_{n}(0)\right|^{2}\right), 1\right)+\left\|\hat{u}_{n}^{\prime}(0)\right\|_{L^{2}}^{2}+\gamma\left\|\nabla \hat{u}_{n}^{\prime}(0)\right\|_{L^{2} \times L^{2}}^{2}\right) .
\end{aligned}
$$

We can then write

$$
\hat{E}_{\gamma}\left(\hat{u}_{n}(t)\right) \leq C_{11} \hat{E}_{\gamma}\left(\hat{u}_{n}(0)\right) e^{-C_{7} t}+C_{12}\|f\|_{\mathcal{V}}^{2}
$$

and, bearing in mind (19),

$$
E_{\gamma}\left(\hat{u}_{n}(t)\right) \leq C_{13} E_{\gamma}\left(\hat{u}_{n}(0)\right) e^{-C_{14} t}+C_{15}\|f\|_{\mathcal{V}}^{2} .
$$

Choosing then the subsequences $\left\{\hat{u}_{n, k}(t)\right\}$ as we made in the proof of Theorem 4, Theorem 5 is proved

Proof of Theorem 6. Let $\hat{u}(t)$ be the solution of problem (12). We can write the variational equation between $t$ and $t+\varepsilon$

$$
\begin{array}{r}
\int_{t}^{t+\varepsilon}\left\{-\left(\hat{u}^{\prime}(\tau), h^{\prime}(\tau)\right)+\delta\left(\hat{u}^{\prime}(\tau), h(\tau)\right)\right. \\
-K(\Delta \hat{u}(\tau), h(\tau))+(\nabla(\mathbf{b}(\nabla \hat{u}(\tau))), h(\tau)) \\
\left.-\gamma\left(\Delta \hat{u}^{\prime}(\tau), h^{\prime}(\tau)\right)-\gamma \delta\left(\Delta \hat{u}^{\prime}(\tau), h(\tau)\right)-(f(\tau), h(\tau))\right\} d \tau \\
+\left[\left(\hat{u}^{\prime}(t), h(t)\right)+\gamma\left(\Delta \hat{u}^{\prime}(t), h(t)\right)\right]_{t_{1}}^{t_{2}}=0 .
\end{array}
$$

Setting $h_{\mu}=J_{\mu}^{2} \hat{u}^{\prime}$, we can observe that $h_{\mu} \in H^{1}\left(0, T ; H^{1}\right)$, and we can then use it as a test function in the above variational equation.

As in [6], one can prove the relations

$$
\begin{aligned}
\lim _{\mu \rightarrow 0} \int_{t}^{t+\varepsilon}\left(\hat{u}^{\prime}(\tau), h_{\mu}^{\prime}(\tau)\right) d \tau & =\frac{1}{2}\left(\left\|\hat{u}^{\prime}(t+\varepsilon)\right\|_{L^{2}}^{2}-\left\|\hat{u}^{\prime}(t)\right\|_{L^{2}}^{2}\right) \\
\lim _{\mu \rightarrow 0} \int_{t}^{t+\varepsilon}\left(\Delta \hat{u}(\tau), h_{\mu}(\tau)\right) d \tau & =-\frac{1}{2}\left(\|\nabla \hat{u}(t+\varepsilon)\|_{L^{2} \times L^{2}}^{2}-\|\nabla \hat{u}(t)\|_{L^{2} \times L^{2}}^{2}\right) \\
\lim _{\mu \rightarrow 0} \int_{t}^{t+\varepsilon}\left(\Delta \hat{u}^{\prime}(\tau), h_{\mu}^{\prime}(\tau)\right) d \tau & =-\frac{1}{2}\left(\left\|\nabla \hat{u}^{\prime}(t+\varepsilon)\right\|_{L^{2} \times L^{2}}^{2}-\left\|\nabla \hat{u}^{\prime}(t)\right\|_{L^{2} \times L^{2}}^{2}\right) \\
\lim _{\mu \rightarrow 0}\left(\hat{u}^{\prime}(\tau), h_{\mu}(\tau)\right) & =\left\|\hat{u}^{\prime}(\tau)\right\|_{L^{2}}^{2}
\end{aligned}
$$


and

$$
\begin{aligned}
\lim _{\mu \rightarrow 0} \int_{t}^{t+\varepsilon} & \left(\nabla(\mathbf{b}(\nabla \hat{u}(\tau))), h_{\mu}(\tau)\right) d \tau \\
= & \int_{t}^{t+\varepsilon}\left((\mathbf{b}(\nabla \hat{u}(\tau))), \nabla \hat{u}^{\prime}(\tau)\right)_{L^{2} \times L^{2}} d \tau .
\end{aligned}
$$

It is then easy to prove that

$$
\lim _{\mu \rightarrow 0}\left(f(\tau), h_{\mu}(\tau)\right)=\left(f(\tau), \hat{u}^{\prime}(\tau)\right) .
$$

Then, recalling (16) we have by the Schwarz inequality

$$
\begin{aligned}
& E_{\gamma}(\hat{u}(t+\varepsilon))-E_{\gamma}(\hat{u}(t)) \\
& \quad \leq \int_{t}^{t+\varepsilon}\left\{\left(\|f(\tau)\|_{L^{2}}-\delta\left\|\hat{u}^{\prime}(\tau)\right\|_{L^{2}}\right)\left\|\hat{u}^{\prime}(\tau)\right\|_{L^{2}}\right. \\
& \left.\quad+\left(\|\mathbf{b}(\nabla \hat{u}(\tau))\|_{L^{2} \times L^{2}}-\gamma \delta\left\|\nabla \hat{u}^{\prime}(\tau)\right\|_{L^{2} \times L^{2}}\right)\left\|\nabla \hat{u}^{\prime}(\tau)\right\|\right\} d \tau .
\end{aligned}
$$

By Theorem 4 we know that $\left\|\hat{u}^{\prime}(t)\right\|_{L^{2}} \leq A_{1}$ and $\left\|\nabla \hat{u}^{\prime}(t)\right\|_{L^{2} \times L^{2}} \leq A_{1}$. Combining this with the usual assumption on $f$, we conclude that there exists a constant $A_{2}>0$ such that

$$
\begin{gathered}
\mid\left\{\left(\|f(\tau)\|_{L^{2}}-\delta\left\|\hat{u}^{\prime}(\tau)\right\|_{L^{2}}\right)\left\|\hat{u}^{\prime}(\tau)\right\|_{L^{2}}\right. \\
\left.+\left(\|\mathbf{b}(\nabla \hat{u}(\tau))\|_{L^{2} \times L^{2}}-\gamma \delta\left\|\nabla \hat{u}^{\prime}(\tau)\right\|_{L^{2} \times L^{2}}\right)\left\|\nabla \hat{u}^{\prime}(\tau)\right\|\right\} \mid \leq A_{2} .
\end{gathered}
$$

Then $\left|E_{\gamma}(\hat{u}(t+\varepsilon))-E_{\gamma}(\hat{u}(t))\right| \leq \nu_{1}(\varepsilon)$ for all $t \in \mathbb{R}^{+}$which proves our theorem

Proof of Theorem 7. We begin recalling that, if the operator $A: H_{0}^{1} \rightarrow$ $\left(H_{0}^{1}\right)^{*}$ is defined by

$$
(A u, h)=\int_{\Omega} a(|\nabla u|) \nabla u \cdot \nabla h d \Omega
$$

and the function $a: \mathbb{R} \rightarrow \mathbb{R}$ is non-decreasing and satisfies

$$
(a(x) x-a(y) y, x-y) \geq \alpha|x-y| \quad(x, y \in \mathbb{R}),
$$

then $A$ is strongly monotone. We can then see that the function $\psi$ of (8) verifies the above conditions. Therefore the operator

$$
A u=\operatorname{div}\left[\left(1+|\nabla u|^{2}\right)^{\frac{1}{2}}-\rho^{2} \frac{\nabla v}{\left(1+|\nabla u|^{2}\right)^{\frac{1}{2}}}\right],
$$


obtained from (1) when $\phi$ is the usual Hooke's law, is strongly monotone. We know then that, if $f \in\left(H_{0}^{1}\right)^{*}$, the equation

$$
\operatorname{div}\left[\left(1+|\nabla u|^{2}\right)^{\frac{1}{2}}-\rho^{2} \frac{\nabla v}{\left(1+|\nabla u|^{2}\right)^{\frac{1}{2}}}\right]=f_{0}
$$

has a unique solution.

Now, let $\hat{u}(t)$ be a solution of equation (12). By Theorem $4, E_{\gamma}(\hat{u}(t))$ is bounded. Then $\hat{E}_{\gamma}(\hat{u}(t))$ is bounded. By Theorem $6, E_{\gamma}(\hat{u}(t))$ is uniformly continuous. Then both $K\|\nabla \hat{u}(t)\|_{L^{2} \times L^{2}}^{2}$ and $\|\hat{u}\|_{L^{2}}^{2}+\gamma\left\|\nabla \hat{u}^{\prime}(t)\right\|_{L^{2} \times L^{2}}^{2}$ are uniformly continuous, and then $\hat{E}_{\gamma}(\hat{u}(t))$ is uniformly continuous.

Acting now as in the proof of Theorem 6, we can write the variational equation between $t=n$ and $t=n+1$ and, as in the proof of Theorem 4 (equation (27)), we get

$$
\begin{aligned}
& \hat{E}_{\gamma}(\hat{u}(n+1))-\hat{E}_{\gamma}(\hat{u}(n)) \\
& \quad=\int_{n}^{n+1}\left\{\left(f(\tau), \hat{u}^{\prime}(\tau)\right)-\delta\left\|\hat{u}^{\prime}(\tau)\right\|_{L^{2}}^{2}-\gamma \delta\left\|\nabla \hat{u}^{\prime}(\tau)\right\|_{L^{2} \times L^{2}}^{2}\right\} d \tau .
\end{aligned}
$$

We know that $f(t)=f_{0}$. Then

$$
\begin{aligned}
& \hat{E}_{\gamma}(\hat{u}(n+1))-\hat{E}_{\gamma}(\hat{u}(n)) \\
& \quad=\left(f_{0}, \hat{u}(n+1)-\hat{u}(n)\right)-\delta \int_{n}^{n+1}\left(\left\|\hat{u}^{\prime}(\tau)\right\|_{L^{2}}^{2}+\gamma\left\|\nabla \hat{u}^{\prime}(\tau)\right\|_{L^{2} \times L^{2}}^{2}\right) d \tau .
\end{aligned}
$$

Thus $\hat{E}_{\gamma}(\hat{u}(n))$ is a bounded sequence, and then we can extract a converging subsequence.

Moreover, as $\{\hat{u}(n)\}$ is a bounded sequence in $H^{2}$, we can extract a subsequence $\left\{\hat{u}\left(n_{k}\right)\right\}$ strongly convergent in $H_{0}^{1}$ and then

$$
\lim _{k \rightarrow+\infty} \int_{n_{k}}^{n_{k+1}}\left(\left\|\hat{u}^{\prime}(\tau)\right\|_{L^{2}}^{2}+\gamma\left\|\nabla \hat{u}^{\prime}(\tau)\right\|_{L^{2} \times L^{2}}^{2}\right) d \tau=0 .
$$

Thus, for all $\varepsilon>0$ there exists $k_{\varepsilon}$ such that

$$
\int_{n_{k_{\varepsilon}}}^{+\infty}\left(\left\|\hat{u}^{\prime}(\tau)\right\|_{L^{2}}^{2}+\gamma\left\|\nabla \hat{u}^{\prime}(\tau)\right\|_{L^{2} \times L^{2}}^{2}\right) d \tau<\varepsilon
$$

as $\left\|\hat{u}^{\prime}(t)\right\|_{L^{2}}^{2}+\gamma\left\|\nabla \hat{u}^{\prime}(t)\right\|_{L^{2} \times L^{2}}^{2}$ is greater than 0 and uniformly continuous. This means that

$$
\lim _{k \rightarrow+\infty}\left\|\hat{u}^{\prime}\left(n_{k}\right)\right\|_{L^{2}}^{2}+\gamma\left\|\nabla \hat{u}^{\prime}\left(n_{k}\right)\right\|_{L^{2} \times L^{2}}^{2}=0 .
$$


We set $v=\lim _{k \rightarrow+\infty} \hat{u}\left(n_{k}\right)$ and define the sequence $\hat{u}_{k}(t)=\hat{u}\left(t+n_{k}\right)$. Then $\lim _{k \rightarrow+\infty} \hat{u}_{k}(t)=u(t)$ strongly in $L^{2}\left(H_{0}^{1}\right)$. We can see each $\hat{u}_{k}(t)$ as a solution of equation (12) with $\hat{u}_{k}^{\prime}(0)=\beta_{k}=u\left(n_{k}\right)$ and $\nabla \hat{u}_{k}^{\prime}(0)=\nabla \beta_{k}$. Then $u(t)$ is the solution corresponding to

$$
\left.\begin{array}{rl}
u(0) & =v \\
u^{\prime}(0)=\lim _{k \rightarrow+\infty} \hat{u}^{\prime}\left(n_{k}\right) & =0 \\
\nabla u^{\prime}(0)=\lim _{k \rightarrow+\infty} \nabla \hat{u}^{\prime}\left(n_{k}\right) & =0
\end{array}\right\} .
$$

If $v$ is the (unique) solution of (30), then $u(t) \equiv v$

Proof of Theorem 8. The proof can be obtained modifying that of Theorem 4. Therefore we omit to write it

Proof of Theorem 9. We can follow the sketch of the proof of Theorem 5 , provided that we substitute in each equation the terms containing $\gamma$ and $\gamma \delta$ with the equivalent terms containing $\eta$ obtained as

$$
\eta\left(\Delta \tilde{u}_{n}(t), \Delta w_{n}(t)\right)=\eta \frac{1}{2} \frac{d}{d t}\left\|\Delta \tilde{u}_{n}(t)\right\|_{L^{2}}^{2}+\varepsilon \eta\left\|\Delta \tilde{u}_{n}(t)\right\|_{L^{2}}^{2} .
$$

Now we can define

$$
\mathcal{U}_{n}(t)=\frac{1}{2}\left[\left(\Psi\left(\left|\nabla \tilde{u}_{n}(t)\right|^{2}\right), 1\right)+\left\|w_{n}(t)\right\|_{L^{2}}^{2}+\eta\left\|\Delta \tilde{u}_{n}(t)\right\|_{L^{2}}^{2}\right]
$$

and find two constants $C_{1}$ and $C_{2}$ such that

$$
\frac{d}{d t} \mathcal{U}_{n}(t)+C_{1} \mathcal{U}_{n}(t) \leq C_{2}\|f(t)\|_{L^{2}} \sqrt{\mathcal{U}_{n}(t)} .
$$

The thesis then follows in an analogous way

Proof of Theorem 10. The thesis follows easily after proving that

$$
\lim _{\mu \rightarrow 0} \int_{t_{1}}^{t_{2}}\left(\Delta w(\tau), \Delta h_{\mu}(\tau)\right) d \tau=\frac{1}{2}\left(\left\|\Delta w\left(t_{2}\right)\right\|_{L^{2}}^{2}-\left\|\Delta w\left(t_{1}\right)\right\|_{L^{2}}^{2}\right) .
$$

This could be done following the similar proofs illustrated in [6]

Proofs of Theorems 11, 12 and 14. The proof of Theorem 11 can be obtained by a combination of the proofs of Theorems 4 and 8. Similarly, the proof of Theorem 12 can be obtained by a combination of the proofs of Theorems 5 and 9, and the proof of Theorem 14 can be obtained by a combination of the proofs of Theorems 6 and 10 
Proof of Theorem 13. We define the sequence $\left\{u_{n}(t)\right\}$ in the following way: if $t \geq-n$, then $u_{n}(t)$ is the solution (which exists, is unique and $E(\cdot)-$ continuous) satisfying the initial conditions $u_{n}(-n)=0, u_{n}^{\prime}(-n)=0$ and $u_{n}(t) \equiv 0$ for $t<-n$. Setting

$$
f_{n}(t)= \begin{cases}f(t) & \text { if } t \geq-n \\ 0 & \text { if } t<-n\end{cases}
$$

we can say that $u_{n}$ is a solution in $-\infty<t<\infty$ corresponding to the known term $f_{n}$.

From Theorem 11 we have that $E\left(u_{n}(t)\right) \leq A_{1}$, where (in this case, as the initial conditions are vanishing) $A_{1}$ depends only on $\|f\|_{\mathcal{V}}^{2}$. By Theorem 14 we obtain $E\left(u_{n}(t+\eta)-u_{n}(t)\right) \rightarrow 0 \quad(\eta \rightarrow 0)$ as $E\left(u_{n}(t)\right)=0$ if $t<-n$. This relation obviously holds also for $t<-n$. Then the $u_{n}$ are uniformly continuous (with respect to the energy norm $E(\cdot)$ ) for $t \in \mathbb{R}$.

We have, thanks to the above uniform limitation, that $E\left(u_{n}(t)\right) \rightarrow E(u(t))$ weakly for all $t \in \mathbb{R}$. Indeed, for all $h \in \mathcal{H}$, we have that the functions $\left(u_{n}(t), h\right)_{\mathcal{H}}$ are equally bounded and equally uniformly continuous for $t \in \mathbb{R}$. The separability of $\mathcal{H}$ guarantees us that the sequence $\left\{\left(u_{n}(t), h\right)_{\mathcal{H}}\right\}$ converges uniformly on every bounded interval. Moreover, we have

$$
E(u(t+\varepsilon)-u(t)) \leq \min \lim _{n \rightarrow \infty} E\left(u_{n}(t+\varepsilon)-u_{n}(t)\right) \nu(\varepsilon) .
$$

Again from $E\left(u_{n}(t)\right) \leq A_{1}$, the functions $u_{n}(t)$ are equally bounded in $H_{0}^{1} \cap H^{2}$ and equally upper continuous in $L^{2}$ for all $t \in \mathbb{R}$. We can then select from $\left\{u_{n}(t)\right\}$ a subsequence, again denoted $\left\{u_{n}(t)\right\}$, such that $u_{n}(t) \rightarrow u(t)$ strongly in $H_{0}^{1}$, uniformly in every bounded interval. Moreover, as the $u_{n}^{\prime}(t)$ are equally upper continuous in $L^{2}$ for all $t \in \mathbb{R}$, we can say that $u_{n}^{\prime}(t) \rightarrow u^{\prime}(t)$ strongly in $L^{2}$, uniformly in every bounded interval. We can conclude then that $u(t)$ is a bounded solution, for $t \in \mathbb{R}$, of (14). Moreover, thanks to (31), $u(t)$ is upper continuous with respect to $E(\cdot)$, for $t \in \mathbb{R}$

\section{References}

[1] Adams, R. A.: Sobolev Spaces. New York: Academic Press 1975.

[2] Prouse, G., Rolandi, F. and A. Zaretti: Sulla corda e verga vibranti. Quad IAC, Serie III, 111 (1977).

[3] Temam, R.: Infinite-Dimensional Dynamical Systems in Mechanics and Physics. New York: Springer-Verlag 1988.

[4] Iannelli, A., Prouse, G. and A. Veneziani: Analysis of a nonlinear model of the vibrating string. NoDEA 3 (1996), $149-177$. 
[5] Pata, V., Prouse, G. and M. I. Vishik: Traveling waves of dissipative nonautonomous hyperbolic equations in a strip. (Adv. in Diff. Equ. 3 (1998), 249 270.

[6] Iannelli, A.: On a nonlinear model of the vibrating membrane. Z. Anal. Anw. 17 (1998), $831-841$.

Received 20.01.2003; in revised form 13.11.2003 